\title{
THE BROAD-LINED Type Ic SN 2012ap AND THE NATURE OF RELATIVISTIC SUPERNOVAE LACKING A GAMMA-RAY BURST DETECTION
}

\author{
D. Milisavljevic ${ }^{1}$, R. Margutti ${ }^{1}$, J. T. Parrent ${ }^{1}$, A. M. Soderberg ${ }^{1}$, R. A. Fesen ${ }^{2}$, P. Mazzali ${ }^{3,4,5}$, K. Maeda ${ }^{6,7}$, \\ N. E. Sanders ${ }^{1}$, S. B. Cenko ${ }^{8,9}$, J. M. Silverman ${ }^{10}$, A. V. Filippenko ${ }^{9}$, A. Kamble $^{1}$, S. Chakrabortit ${ }^{1}$, M. R. Drout ${ }^{1}$, \\ R. P. Kirshner ${ }^{1}$, T. E. Pickering ${ }^{11,12}$, K. Kawabata ${ }^{13}$, T. Hattori ${ }^{14}$, E. Y. HsiaO ${ }^{15}, 16$, M. D. Stritzinger ${ }^{16}$, \\ G. H. MARION ${ }^{10}$, J. VinKo ${ }^{10,17}$, AND J. C. WheELER ${ }^{10}$ \\ ${ }^{1}$ Harvard-Smithsonian Center for Astrophysics, 60 Garden Street, Cambridge, MA 02138, USA; dmilisav@cfa.harvard.edu \\ ${ }^{2}$ Department of Physics and Astronomy, Dartmouth College, 6127 Wilder Laboratory, Hanover, NH 03755, USA \\ ${ }^{3}$ Astrophysics Research Institute, Liverpool John Moores University, Liverpool L3 5RF, UK \\ ${ }^{4}$ Max-Planck-Institut für Astrophysik, Karl-Schwarzschild-Strasse 1, D-85748 Garching, Germany \\ ${ }^{5}$ INAF-Osservatorio Astronomico di Padova, Vicolo dell'Osservatorio 5, I-35122, Padova, Italy \\ ${ }^{6}$ Department of Astronomy, Kyoto University, Kitashirakawa-Oiwake-cho, Sakyo-ku, Kyoto 606-8502, Japan \\ ${ }^{7}$ Kavli Institute for the Physics and Mathematics of the Universe (WPI), Todai Institutes for Advanced Study, University of Tokyo, \\ 5-1-5 Kashiwanoha, Kashiwa, Chiba 277-8583, Japan \\ ${ }^{8}$ Astrophysics Science Division, NASA Goddard Space Flight Center, Mail Code 661, Greenbelt, MD 20771, USA \\ ${ }^{9}$ Department of Astronomy, University of California, Berkeley, CA 94720-3411, USA \\ ${ }^{10}$ University of Texas at Austin, 1 University Station C1400, Austin, TX 78712-0259, USA \\ ${ }^{11}$ Southern African Large Telescope, P.O. Box 9, Observatory 7935, Cape Town, South Africa \\ ${ }^{12}$ Space Telescope Science Institute, 3700 San Martin Drive, Baltimore, MD 21218, USA \\ ${ }^{13}$ Hiroshima Astrophysical Science Center, Hiroshima University, Higashi-Hiroshima, Hiroshima 739-8526, Japan \\ ${ }^{14}$ Subaru Telescope, National Astronomical Observatory of Japan, Hilo, HI 96720, USA \\ ${ }^{15}$ Carnegie Observatories, Las Campanas Observatory, Colina El Pino, Casilla 601, Chile \\ ${ }^{16}$ Department of Physics and Astronomy, Aarhus University, Ny Munkegade, DK-8000 Aarhus C, Denmark \\ ${ }^{17}$ Department of Optics and Quantum Electronics, University of Szeged, Dom ter 9, 6720 Szeged, Hungary \\ Received 2014 August 6; accepted 2014 November 6; published 2015 January 15
}

\begin{abstract}
We present ultraviolet, optical, and near-infrared observations of SN 2012ap, a broad-lined Type Ic supernova in the galaxy NGC 1729 that produced a relativistic and rapidly decelerating outflow without a gamma-ray burst signature. Photometry and spectroscopy follow the flux evolution from -13 to +272 days past the $B$-band maximum of $-17.4 \pm 0.5 \mathrm{mag}$. The spectra are dominated by Fe II, O I, and Ca II absorption lines at ejecta velocities of $v \approx$ $20,000 \mathrm{~km} \mathrm{~s}^{-1}$ that change slowly over time. Other spectral absorption lines are consistent with contributions from photospheric He I, and hydrogen may also be present at higher velocities $\left(v \gtrsim 27,000 \mathrm{~km} \mathrm{~s}^{-1}\right)$. We use these observations to estimate explosion properties and derive a total ejecta mass of $\sim 2.7 M_{\odot}$, a kinetic energy of $\sim 1.0 \times 10^{52} \mathrm{erg}$, and a ${ }^{56} \mathrm{Ni}$ mass of $0.1-0.2 M_{\odot}$. Nebular spectra $(t>200$ days $)$ exhibit an asymmetric double-peaked [OI] $\lambda \lambda 6300,6364$ emission profile that we associate with absorption in the supernova interior, although toroidal ejecta geometry is an alternative explanation. SN 2012ap joins SN 2009bb as another exceptional supernova that shows evidence for a central engine (e.g., black hole accretion or magnetar) capable of launching a non-negligible portion of ejecta to relativistic velocities without a coincident gamma-ray burst detection. Defining attributes of their progenitor systems may be related to notable observed properties including environmental metallicities of $Z \gtrsim Z_{\odot}$, moderate to high levels of host galaxy extinction $(E(B-V)>0.4 \mathrm{mag})$, detection of high-velocity helium at early epochs, and a high relative flux ratio of $[\mathrm{Ca} \mathrm{II}] /\left[\mathrm{O}_{\mathrm{I}}\right]>1$ at nebular epochs. These events support the notion that jet activity at various energy scales may be present in a wide range of supernovae.
\end{abstract}

Key words: gamma-ray burst: general - supernovae: general - supernovae: individual (SN 2012ap)

\section{INTRODUCTION}

The spectral features of core-collapse supernovae $(\mathrm{SNe})$ provide a basis of classification that reflects properties of their progenitor stars and explosion dynamics (Minkowski 1941; Shklovskii 1960; Kirshner et al. 1973; Oke \& Searle 1974). By standard definition, Type Ib SNe lack conspicuous absorptions attributable to hydrogen, and Type Ic SNe lack conspicuous absorptions attributable to hydrogen and helium (Filippenko 1997; Matheson et al. 2001; Turatto 2003; Modjaz et al. 2014). These two subgroups, however, may have many deviant cases (e.g., Branch et al. 2006; Parrent et al. 2007; James \& Baron 2010), and a possible continuum between them is sometimes acknowledged by using the designation Type Ibc (hereafter SN Ibc).
SN Ibc are thought to originate from stars that have been largely stripped of their outer envelopes (Wheeler et al. 1987; Clocchiatti et al. 1997), via radiative winds (Woosley et al. 1993) or various forms of binary interaction (Podsiadlowski et al. 1992; Nomoto et al. 1995). No secure direct identification has yet been made of a SN Ibc progenitor system (Van Dyk et al. 2003; Smartt 2009; Eldridge et al. 2013), with the possible exception of iPTF13bvn (Cao et al. 2013; Groh et al. 2013; Bersten et al. 2014; Eldridge et al. 2014; Fremling et al. 2014).

Broad-lined Type Ic SNe (hereafter SN Ic-bl) are a subset of SN Ibc that show exceptionally high expansion velocities in their bulk ejecta reaching $\sim 0.1 c$. Generally, SN Ic-bl are associated with large kinetic energies (several $10^{52} \mathrm{erg}$ ) approximately 10 times those of normal SN Ibc, and ejected masses of several $M_{\odot}$, of which $\sim 0.5 M_{\odot}$ is ${ }^{56} \mathrm{Ni}$ (Mazzali et al. 2008a). 
However, the handful of SN Ic-bl known are diverse and can vary considerably in these explosion properties (Nomoto et al. 2007). This diversity has been underscored by recent examples such as SN 2007bg (Young et al. 2010), SN 2007ru (Sahu et al. 2009), SN 2009nz (Berger et al. 2011), SN 2010ay (Sanders et al. 2012b), PTF10qts (Walker et al. 2014), SN 2010ah (Corsi et al. 2011; Mazzali et al. 2013), and PTF12gzk (Ben-Ami et al. 2012).

A crucial and revealing aspect of SN Ic-bl is that they can accompany long-duration gamma-ray bursts (GRBs). The coincidence of nearby GRBs with SNe including GRB980425 with SN 1998bw (Galama et al. 1998) and GRB030329 with SN 2003dh (Stanek et al. 2003; Matheson et al. 2003) has established that all well-observed GRB-SN are SN Ic-bl. However, as demonstrated by objects such as SN 2002ap (Mazzali et al. 2002; Berger et al. 2002), the converse is not true: namely, not all SN Ic-bl are associated with GRBs.

It is an open question as to why some SN Ic-bl are associated with GRBs and others are not. Radio observations seem to rule out the possibility that all SN Ic-bl without a GRB detection are off-axis GRBs (Soderberg et al. 2006b). Thus, certain properties of the progenitor systems and explosion dynamics of these $\mathrm{SNe}$ must dictate why some explosions are not sufficient to generate a GRB. Notable properties include the following. (1) SN Ic-bl not associated with GRBs tend to have smaller values of ejecta mass, explosion energy, and luminosity as compared to the GRB-SN (Nomoto et al. 2007)—although exceptions do exist, such as the relatively subluminous SN 2010bh associated with GRB 100316D (Chornock et al. 2010; Olivares E. et al. 2012; Bufano et al. 2012). (2) The relative rates of GRB and SN Ic-bl are comparable $\left(10^{-6}-10^{-5} \mathrm{yr}^{-1}\right.$ per galaxy) and support the notion that they originate from the same population (Podsiadlowski et al. 2004). (3) As with GRBs, SN Ic-bl preferentially occur in regions of high star-formation rates and/or very young stellar populations having subsolar metallicity environments when compared to normal SN Ibc (Kelly \& Kirshner 2012; Sanders et al. 2012a).

Discovery of SN 2009bb provided strong evidence of a continuum between GRB-SN and SN Ic-bl. SN 2009bb was a SN Ic-bl that exhibited radio properties consistent with a nonnegligible portion of its ejecta moving at relativistic speeds as observed in GRBs, yet was subenergetic by a factor of $\sim 100$ and did not have a GRB detection (Soderberg et al. 2010; Pignata et al. 2011; Chakraborti \& Ray 2011). Because the bulk explosion parameters of SN 2009bb could not account for the copious energy coupled to relativistic ejecta, it was concluded that a central engine (e.g., black hole accretion or magnetar) must have driven part of the explosion.

A second and more recent example of this type of event is SN 2012ap. As in SN 2009bb, SN 2012ap was found to have relativistic outflow but without an observed GRB (Chakraborti et al. 2014). Moreover, neither object showed evidence for luminous X-ray emission at late times $(t>10$ days $)$, which sets them apart from subenergetic GRBs (Margutti et al. 2014). These shared properties led Margutti et al. (2014) to conclude that this distinct class of objects may represent the weakest engine-driven explosions, where the central engine is unable to power a successful jet breakout.

Here we report on ultraviolet (UV), optical, and near-infrared observations of SN 2012ap from -13 to +272 days past $B$ band maximum. In Section 2 we discuss the discovery and classification of SN2012ap. Section 3 presents the data, a portion of which have already been published by Milisavljevic

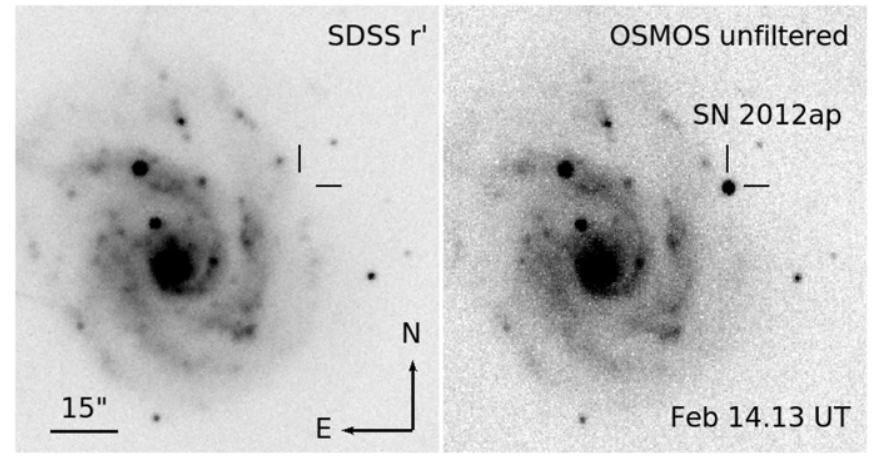

Figure 1. Images of SN 2012ap and its host galaxy NGC 1729. Left: preexplosion SDSS $r^{\prime}$-band image of NGC 1729 with the location of SN 2012ap marked. Right: unfiltered image obtained with the $2.4 \mathrm{~m}$ Hiltner telescope using the OSMOS instrument and MDM4k detector.

et al. (2014, hereafter M14). These data are then used in Section 4 to examine the flux evolution of the SN, reconstruct its bolometric light curve, and derive explosion parameters. In Section 5 we discuss the implications our results and analyses have for potential progenitor systems of SN 2009bb and SN 2012ap. Section 6 concludes with a review of the properties of relativistic SN Ic-bl without a GRB detection and speculates on the extent to which jet activity at various energy scales may be occurring in a wide range of SN.

\section{DISCOVERY AND CLASSIFICATION}

SN 2012ap was first detected by the Lick Observatory Supernova Search (Filippenko et al. 2001) with the $0.76 \mathrm{~m}$ Katzman Automatic Imaging Telescope (KAIT) at coordinates $\alpha(\mathrm{J} 2000)=05^{\mathrm{h}} 00^{\mathrm{m}} 13^{\mathrm{s}} .72$ and $\delta(\mathrm{J} 2000)=-03^{\circ} 20^{\prime} 51^{\prime \prime} .2$ in NGC 1729 on February 10.23 (UT dates are used throughout this paper; Jewett et al. 2012). In Figure 1, pre- and post-explosion images are shown, highlighting the location of the $\mathrm{SN}$ with respect to NGC 1729. The SN is located some $7.1 \mathrm{kpc}$ in projection from the nucleus of the host galaxy along the outer periphery of a spiral arm.

Xu et al. (2012) obtained optical spectra of SN 2012ap on February 11 and 12 with the Chinese Gao-Mei-Gu telescope and classified it as a SN Ibc at early phases. They noted a close similarity with the SN Ib 2008D two weeks before maximum light. Milisavljevic et al. (2012) reported on spectra obtained February 21.8 showing similarities with the broad-lined SN 1998bw (Patat et al. 2001), SN 2002ap (Foley et al. 2003), and the transitional SN 2004aw (Taubenberger et al. 2006) approximately one to two weeks after maximum light. These later spectra were in general agreement with the earlier observations reported by Xu et al., but they no longer showed a strong likeness to SN 2008D.

\section{OBSERVATIONS}

\subsection{Distance and Reddening}

The distance to NGC 1729 estimated by Tully-Fisher measurements is $43 \mathrm{Mpc}$, corresponding to a distance modulus of $\mu=33.17 \pm 0.48 \mathrm{mag}$ (Springob et al. 2009). Conspicuous $\mathrm{Na}$ I D absorption at the rest wavelength of the host galaxy in our spectra (see Section 3.3) and a low apparent brightness at maximum light (Section 3.2) both suggest moderate extinction toward the $\mathrm{SN}$. The foreground Galactic extinction is $E(B-V)_{\text {Galactic }}=0.045 \mathrm{mag}$ (Schlafly \& Finkbeiner 2011). An estimate of the host extinction was made using the equivalent 
Table 1

KAIT Photometry (mag) of SN 2012ap

\begin{tabular}{lcccc}
\hline \hline MJD & $B$ & $V$ & $R$ & $I$ \\
\hline 55967.25 & $\ldots$ & 17.460 .08 & 17.230 .10 & $\ldots$ \\
55973.15 & 17.770 .04 & 16.740 .03 & 16.440 .04 & 16.000 .04 \\
55974.19 & 17.650 .03 & 16.770 .02 & 16.360 .02 & 16.000 .02 \\
55978.15 & 17.880 .04 & 16.760 .01 & 16.290 .02 & 15.850 .02 \\
55980.13 & 18.150 .06 & 16.780 .03 & 16.350 .02 & 15.760 .02 \\
55980.17 & $\ldots$ & $\ldots$ & 16.360 .04 & $\ldots$ \\
55981.15 & 18.320 .08 & 16.900 .03 & 16.350 .03 & 15.750 .04 \\
55982.14 & 18.320 .07 & 16.940 .04 & 16.430 .04 & 15.770 .06 \\
55983.14 & 18.390 .07 & 16.980 .03 & 16.440 .02 & 15.780 .02 \\
55990.14 & 18.970 .18 & 17.560 .06 & 16.810 .04 & 16.100 .02 \\
55990.18 & $\ldots$ & $\ldots$ & 16.810 .08 & $\ldots$ \\
55991.14 & 18.910 .10 & 17.650 .05 & 16.940 .04 & 16.180 .05 \\
55992.16 & 19.120 .28 & 17.630 .07 & 16.960 .04 & 16.270 .04 \\
55993.14 & 18.950 .22 & 17.800 .07 & 17.020 .05 & 16.210 .05 \\
55994.15 & 19.160 .29 & 17.890 .13 & 17.140 .08 & 16.290 .05 \\
55995.16 & 19.550 .18 & 17.930 .06 & 17.110 .03 & 16.310 .03 \\
55996.15 & 19.510 .13 & 17.920 .05 & 17.200 .03 & 16.410 .03 \\
55998.19 & 19.400 .19 & 18.060 .14 & $\ldots$ & 16.510 .06 \\
& & & &
\end{tabular}

Table 2

Swift-UVOT Photometry (mag) of SN 2012ap

\begin{tabular}{lcccc}
\hline \hline MJD & $w 1$ & $u$ & $b$ & $v$ \\
\hline 55969.26 & 19.300 .23 & 18.300 .14 & 17.810 .08 & 17.050 .08 \\
55971.24 & 18.880 .12 & 17.910 .09 & 17.650 .06 & 16.900 .07 \\
55973.41 & 19.060 .13 & 17.920 .09 & 17.520 .06 & 16.770 .06 \\
55975.46 & 19.170 .14 & 18.010 .09 & 17.660 .06 & 16.720 .06 \\
55977.05 & 19.130 .21 & 18.210 .15 & 17.730 .09 & 16.660 .09 \\
55979.72 & 19.430 .17 & 18.420 .12 & 17.870 .07 & 16.850 .07 \\
55983.60 & 19.690 .20 & 18.770 .14 & 18.160 .08 & 16.860 .07 \\
55985.64 & 19.760 .21 & 19.040 .17 & 18.440 .09 & 17.000 .07 \\
55987.62 & 20.270 .72 & 19.430 .52 & 18.270 .18 & 17.130 .18 \\
55988.55 & 20.020 .27 & 19.020 .18 & 18.670 .11 & 17.380 .09
\end{tabular}

width (EW) of the Na I D line. Following Turatto et al. (2003), $\mathrm{EW}(\mathrm{NaI}) \approx 1.2 \AA$ yields estimates of $E(B-V)$ between 0.182 and $0.572 \mathrm{mag}$. Using the same measurement and following instead Poznanski et al. (2012), the estimate is $0.36 \pm 0.07 \mathrm{mag}$. The mean of $\sim 0.4 \mathrm{mag}$ has been used in this paper. Combining the Galactic extinction with the inferred host extinction, a total extinction of $E(B-V)_{\text {total }}=0.45 \mathrm{mag}$ has been adopted. All extinction corrections made in this paper use $E(B-V)_{\text {total }}$, in combination with the standard reddening law of Cardelli et al. (1989) assuming $R_{\mathrm{V}}=3.1$.

Conspicuous narrow absorption features associated with diffuse interstellar bands (DIBs) at rest wavelengths of $4428 \AA$, $5780 \AA$, and $6283 \AA$ are observed in the optical spectra of SN 2012ap, and they change in EW strength between epochs of observations (see M14 for details). In some settings, DIBs have been used to infer the amount of foreground extinction because their EW can be linearly proportional to the amount of foreground reddening (Herbig 1995; Friedman et al. 2011). However, the unusually strong DIB absorptions observed in SN 2012ap are well outside the reliable limits of these relationships and cannot be used to calibrate the extinction.

\subsection{UV/Optical Photometry}

Optical photometry was obtained with KAIT, and both optical and UV photometry was taken with the Swift spacecraft (Gehrels et al. 2004) using the UVOT instrument (Roming et al. 2005). The observed magnitudes are presented in Tables 1 and 2,

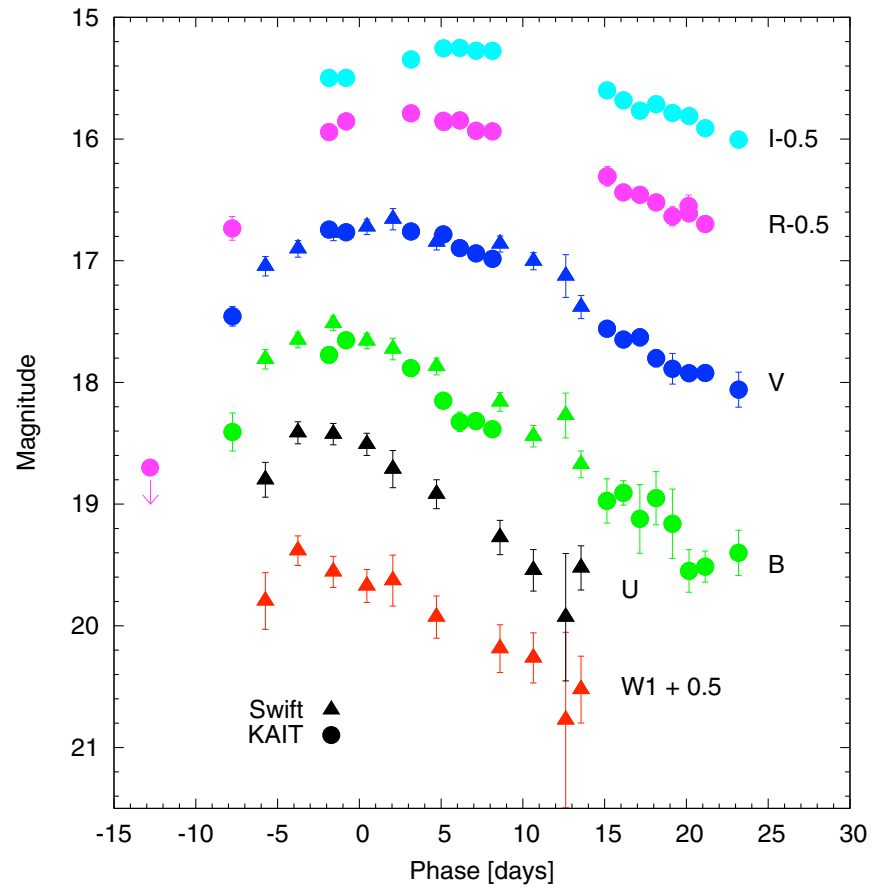

Figure 2. KAIT and Swift UV/optical photometry of SN 2012ap. Phase is with respect to $B$-band maximum on February 18.2 UT.

respectively. KAIT photometry was calibrated using Sloan Digital Sky Survey (SDSS) point sources in the field, and photometric transformations were made from Jordi et al. (2006) to put SDSS photometry into the BVRI system. We analyzed the Swift-UVOT photometric data following the prescriptions of Brown et al. (2009). A $3^{\prime \prime}$ aperture was used to maximize the signal-to-noise ratio $(\mathrm{S} / \mathrm{N})$. Unreported are observations in the $u v w 2$ and $u v m 2$ filters where the $\mathrm{SN}$ was not detected with a significant S/N. Swift-UVOT photometry is based on the photometric system described by Breeveld et al. (2011). In this system, the Swift $b$ and $v$ filters are roughly equivalent to the standard Johnson/Kron-Cousins $B$ and $V$ filters (see Poole et al. 2008 for details), although the difference introduces a small offset in reported magnitudes between the Swift and KAIT photometry. Figure 2 shows the combined Swift-UVOT and KAIT light curves.

\subsection{Optical and Near-infrared Spectra}

Fourteen epochs of long-slit optical spectra of SN 2012ap were obtained from a variety of telescopes and instruments. A single near-infrared spectrum was also obtained. Early-time spectra are shown in Figures 3 (optical) and 4 (near-infrared), and late-time spectra are shown in Figure 5. Table 3 lists the details of the observations.

Many observations were made with the $10 \mathrm{~m}$ Southern African Large Telescope (SALT) at South African Astronomical Observatory using the Robert Stobie Spectrograph (RSS; Burgh et al. 2003). Additional supporting observations came from the $9.2 \mathrm{~m}$ Hobby-Eberly Telescope (HET) using the Marcario LowResolution Spectrograph (LRS; Hill et al. 1998), the 6.5 m MMT telescope using the Blue Channel instrument (Schmidt et al. 1989), the $8.2 \mathrm{~m}$ Subaru Telescope using the Faint Object Camera and Spectrograph (FOCAS; Kashikawa et al. 2002), the $6.5 \mathrm{~m}$ Magellan Baade telescope using the Inamori Magellan Areal Camera and Spectrograph (IMACS; Bigelow et al. 1998), 


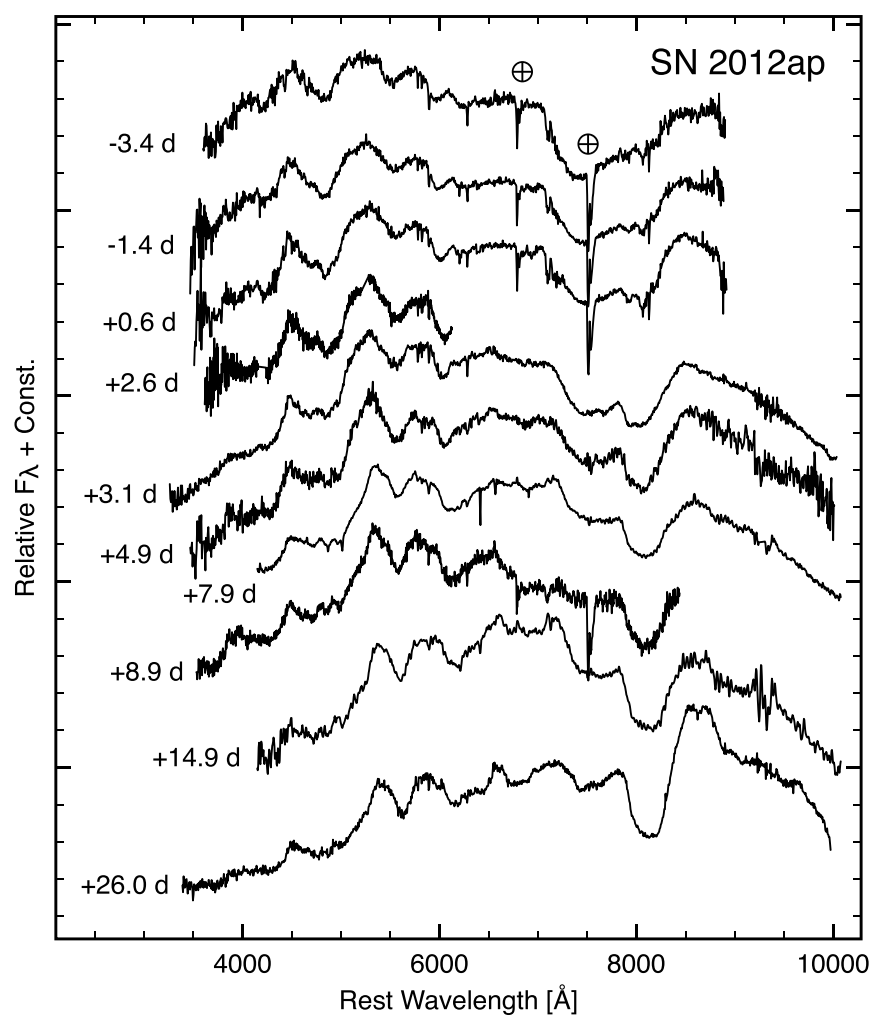

Figure 3. Optical spectra of SN 2012ap. The spectra have been corrected for a redshift of 0.0121 . The " $\oplus$ " symbol shows regions of the spectra that in some cases are contaminated by night-sky $\mathrm{O} 2$ absorption bands.

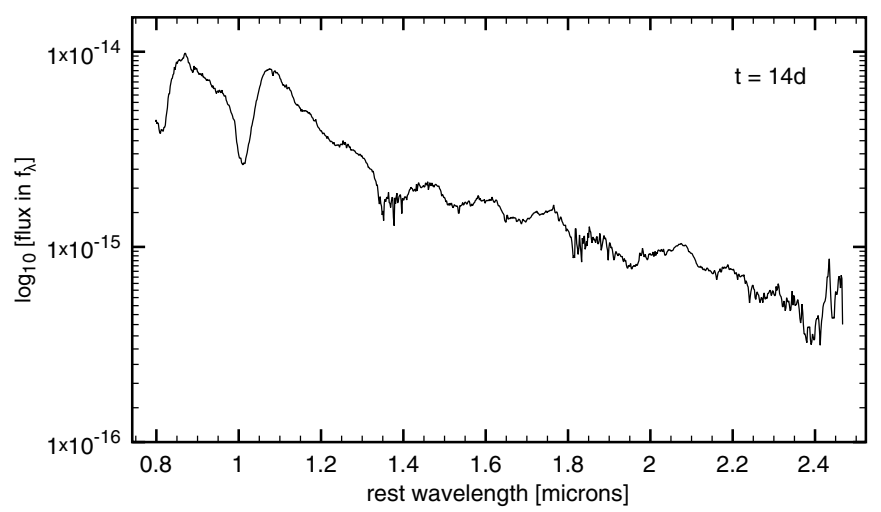

Figure 4. Near-infrared spectrum of SN 2012ap obtained on 2012 March 3 with the FIRE spectrograph on the $6.5 \mathrm{~m}$ Magellan Baade Telescope.

the $10 \mathrm{~m}$ Keck Telescopes using the Low-Resolution Imaging Spectrometer (LRIS; Oke et al. 1995) and the DEep Imaging Multi-Object Spectrograph (DEIMOS; Faber et al. 2003), and the Shane $3 \mathrm{~m}$ Telescope using the Kast double spectrograph (Miller \& Stone 1993). The single near-infrared observation was obtained with the Magellan $6.5 \mathrm{~m}$ Baade Telescope using the FoldedPort Infrared Echellette (FIRE; Simcoe et al. 2008).

Reduction of all optical spectra followed standard procedures using the IRAF/PyRAF software. SALT data were first processed by the PySALT ${ }^{18}$ pipeline (Crawford et al. 2010). Wavelength calibrations were made with arc lamps and verified with the night-sky lines. Relative flux calibrations were made with observations of spectrophotometric standard stars

\footnotetext{
18 http://pysalt.salt.ac.za/
}

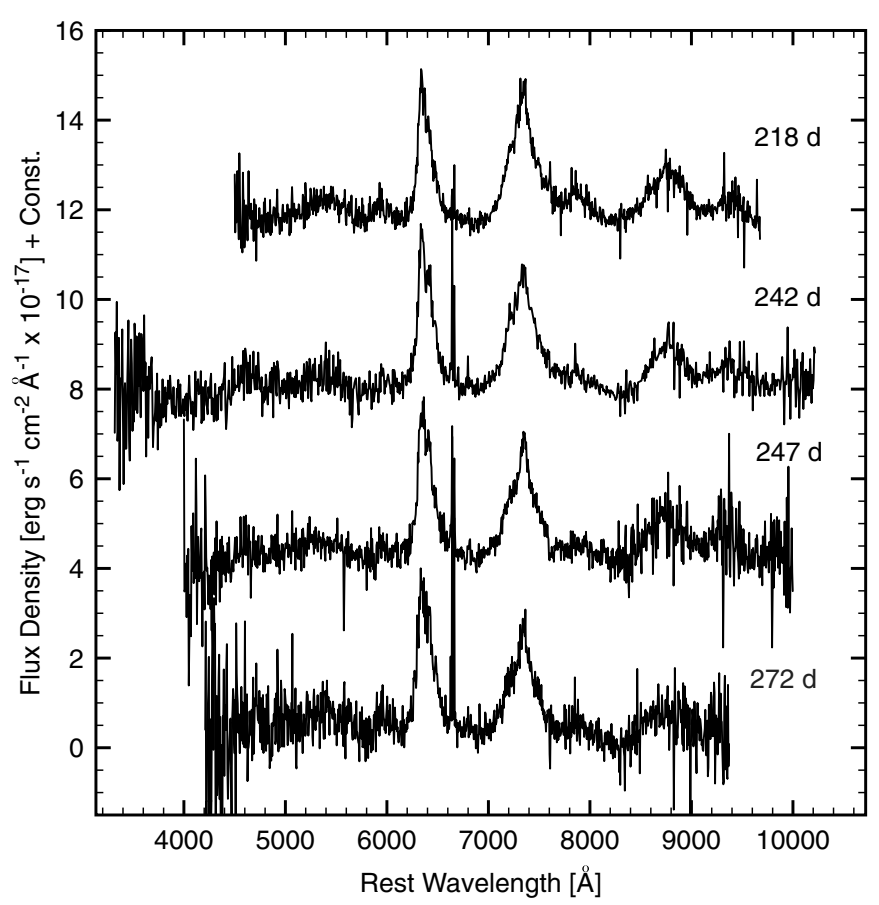

Figure 5. Late-time optical spectra of SN 2012ap during nebular epochs when emission is dominated by forbidden transitions.

Table 3

Summary of Spectroscopic Observations

\begin{tabular}{lccc}
\hline \hline $\begin{array}{l}\text { Date } \\
\text { (UT) }\end{array}$ & MJD & $\begin{array}{c}\text { Phase }^{\mathrm{a}} \\
\text { (days) }\end{array}$ & $\begin{array}{c}\text { Telescope/ } \\
\text { Instrument }\end{array}$ \\
\hline 2012 Feb 14.79 & 55971.79 & -3.4 & SALT/RSS \\
2012 Feb 16.78 & 55973.78 & -1.4 & SALT/RSS \\
2012 Feb 18.80 & 55975.80 & +0.6 & SALT/RSS \\
2012 Feb 20.83 & 55977.83 & +2.6 & SALT/RSS \\
2012 Feb 21.27 & 55978.27 & +3.1 & Keck/LRIS \\
2012 Feb 23.18 & 55980.18 & +4.9 & Lick/Kast \\
2012 Feb 26.10 & 55983.10 & +7.9 & HET/LRS \\
2012 Feb 27.11 & 55984.11 & +8.9 & MMT/Blue Channel \\
2012 Mar 03.03 & 55989.03 & +13.9 & Magellan/FIRE \\
2012 Mar 04.08 & 55990.08 & +14.9 & HET/LRS \\
2012 Mar 15.23 & 56001.23 & +26.0 & Keck/LRIS \\
2012 Sep 23.49 & 56193.49 & +218.3 & Keck/DEIMOS \\
2012 Oct 17.62 & 56217.62 & +242.4 & Keck/LRIS \\
2012 Oct 23.48 & 56223.48 & +248.3 & Subaru/FOCAS \\
2012 Nov 16.31 & 56247.31 & +272.1 & Magellan/IMACS \\
\hline
\end{tabular}

Note. ${ }^{\text {a }}$ Phase is with respect to estimated $B$-band maximum on February 18.2 (MJD 55975.2).

from Oke (1990) and Hamuy et al. (1992, 1994). Gaps between CCD chips have been interpolated in instances when dithering between exposures was not possible, and cosmetic defects have been cleaned manually. In cases when a spectrophotometric standard star could be observed at the time of observation and at comparable airmass, telluric features have been corrected. Near-infrared data were reduced following standard procedures (Hsiao et al. 2013) using a custom-developed IDL pipeline (FIREHOSE).

Spectra have been corrected for a redshift of $z=0.0121$ measured from narrow $\mathrm{H}$ II region lines of [O III] $\lambda \lambda 4959,5007$, $\mathrm{H} \alpha$, and $\left[\mathrm{N}_{\mathrm{II}}\right] \lambda \lambda 6548,6583$ observed near the location of the $\mathrm{SN}$. This value is in agreement with a previous measurement 
Table 4

SN 2012ap Epochs of Maximum Light and Peak Magnitude

\begin{tabular}{lclc}
\hline \hline Filter & $\begin{array}{c}\text { Peak Time } \\
(\text { UT })\end{array}$ & $\begin{array}{c}\text { Peak Time } \\
\text { MJD }\end{array}$ & Peak Mag \\
\hline$B$ & 2012 Feb 18.2 & $55975.2 \pm 0.5$ & $17.63 \pm 0.07$ \\
$V$ & 2012 Feb 19.4 & $55976.4 \pm 0.5$ & $16.72 \pm 0.05$ \\
$R$ & 2012 Feb 19.8 & $55976.8 \pm 0.75$ & $16.28 \pm 0.07$ \\
$I$ & 2012 Feb 26.3 & $55983.3 \pm 0.75$ & $15.74 \pm 0.06$ \\
\hline
\end{tabular}

reported by Theureau et al. (1998) using radio H I lines from the host NGC 1729.

\section{RESULTS}

\subsection{Properties of the UV/Optical Light Curves}

Table 4 shows the properties of our light curves as determined with low-order polynomial fits. The peak in the $B$ band corresponds to an absolute magnitude of $M_{B}=-17.4 \pm 0.5$. This value is relatively faint compared to the majority of SN Ic-bl (Drout et al. 2011; Bianco et al. 2014), being well below that of SN 1998bw $\left(M_{B}=-18.8\right.$ mag; Patat et al. 2001) but above that of SN 2002ap ( $M_{B}=-16.3 \mathrm{mag}$; Foley et al. 2003).

A KAIT image of the region around SN 2012ap was taken February 5.21 prior to detection with limiting $R$-band magnitude of 18.7. This nondetection sets a constraint on the explosion date, with some uncertainty caused by the steepness of the unobserved light curve as it rises and a possible offset owing to a dark phase in the early stages of the explosion (Nakar \& Sari 2012). We used the light curve of SN 2009bb as a template and scaled its peak and width to follow that of SN 2012ap. From this we estimate the explosion date to be February 5, with an uncertainty of $\sim 2$ days, and derive a rise time to peak maximum $B$-band light of 13 days based on the $B$-band maximum on February 18.2.

The $B-V$ color evolution of SN2012ap is illustrated in Figure 6. Also shown are the color evolutions of other SN Ic and Ic-bl. Within the uncertainties of our photometry, the color evolution of SN 2012ap is broadly consistent with those observed in previous SN Ibc. We also find consistency in the $V-R$ colors of SN2012ap with other SN Ibc at similar epochs presented by Drout et al. (2011). The general agreement demonstrates that the adopted $E(B-V)$ value is reasonable.

The extinction-corrected Swift-UVOT and KAIT photometry was used to create a quasi-bolometric light curve ( $L_{\text {bol }}^{\text {quasi }}$ ) of SN 2012ap. The total UV+BVRI flux was determined by summing the integrated fluxes of the different filters with passbands defined in Breeveld et al. (2011) and Jordi et al. (2006, and references therein). Low-order polynomials have been used to interpolate values, and uncertainties have been propagated following standard practice (see, e.g., Margutti et al. 2012; Milisavljevic et al. 2013).

The quasi-bolometric UV+BVRI light curve was transformed into a bolometric light curve assuming $L_{\text {bol }}^{\text {quasi }} \approx 0.8 L_{\text {bol }}$, and that $0.2 L_{\text {bol }}$ is emitted as unobserved near-infrared emission. These assumptions follow from observed properties of SN 2009bb (Pignata et al. 2011) and are in general agreement with those observed in other SN Ic-bl (Valenti et al. 2008a). The bolometric light curve is shown in Figure 7. It is worth noting that outside of SN 2002ap, SN 2012ap is among the least luminous known SN Ic-bl.

We modeled the bolometric light curve to derive the ejecta mass $\left(M_{\mathrm{ej}}\right)$, the nickel mass $\left(M_{\mathrm{Ni}}\right)$, and the kinetic energy of the

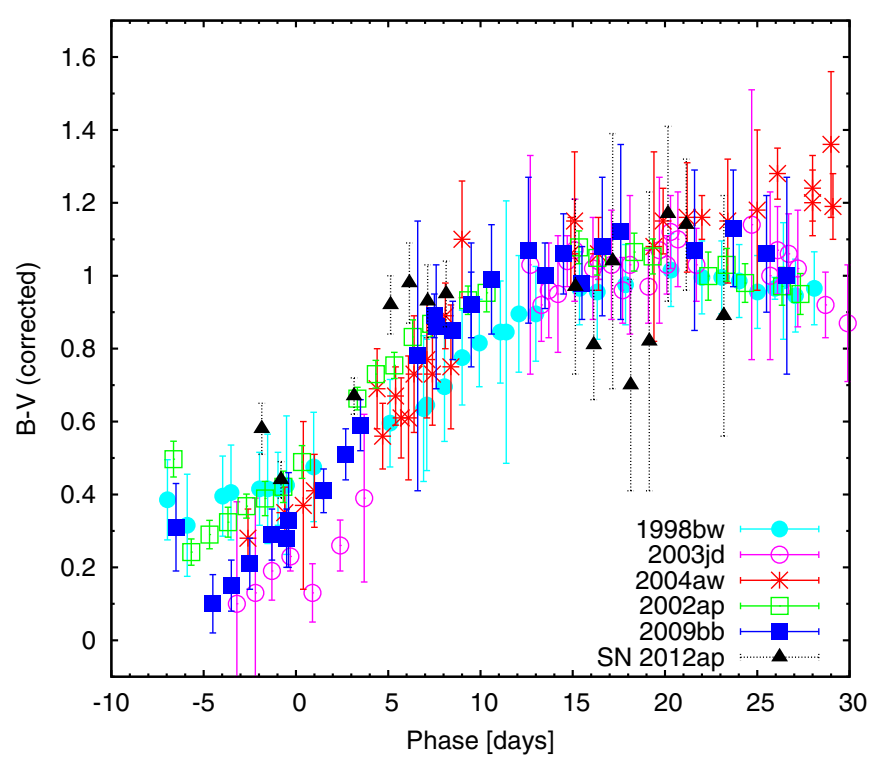

Figure 6. Color evolution of SN 2012ap compared to that of various SN Ic and Ic-bl. References for data on individual supernovae and adopted $E(B-V)$ values are as follows: SN 1998bw (0.0645 mag; Clocchiatti et al. 2011), SN 2003jd (0.14 mag; Valenti et al. 2008a), SN 2004aw (0.37 mag; Taubenberger et al. 2006), SN 2002ap (0.079 mag; Foley et al. 2003), SN $2009 \mathrm{bb}$ (0.58 mag; Pignata et al. 2011), SN 2012ap (0.45 mag; this paper).

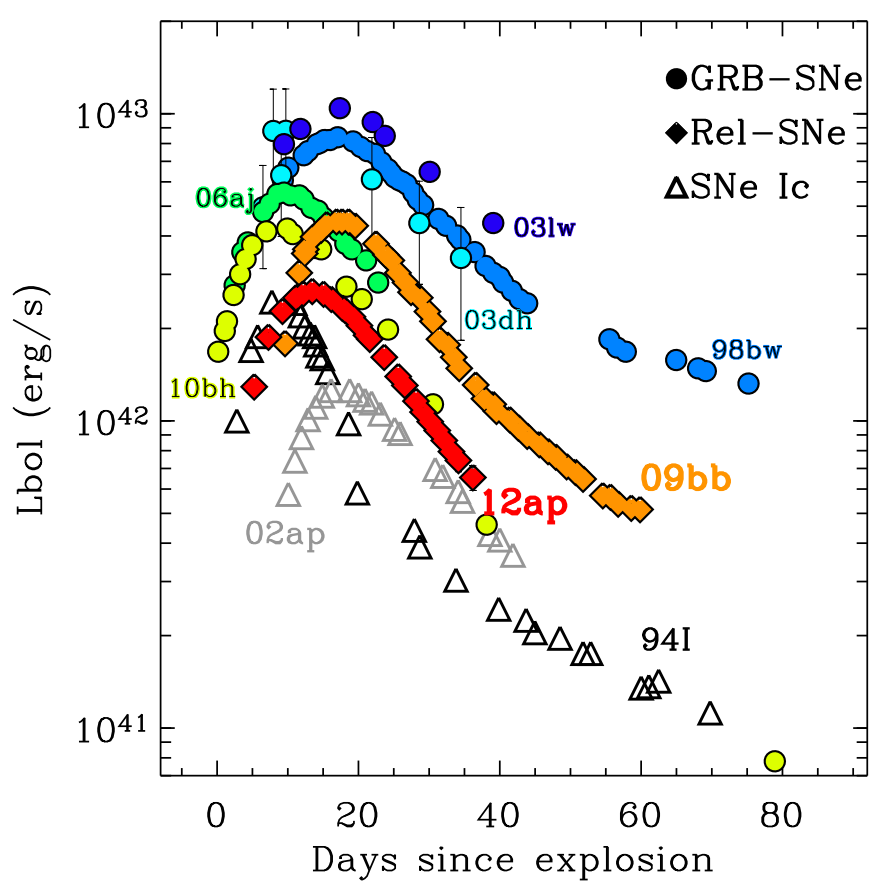

Figure 7. Bolometric light curve of SN2012ap. Data for all other objects are obtained from Olivares E. et al. (2012), except for SN 2003dh, which is from Pian et al. (2006). Error bars shown for SN 2012ap are comparable to the symbol size.

ejecta $\left(E_{\mathrm{k}}\right)$ following the procedures of Valenti et al. (2008a) and Wheeler et al. (2014) that are based on the formalism outlined by Arnett (1982). We assumed that the early-time $\left(\Delta t_{\text {exp }}<30\right.$ days $)$ light curve corresponded with the photospheric regime, and that the late-time ( $\Delta t_{\text {exp }}>30$ days) light curve corresponded with the nebular regime when the optical depth of the ejecta decreases and the observed luminosity is 

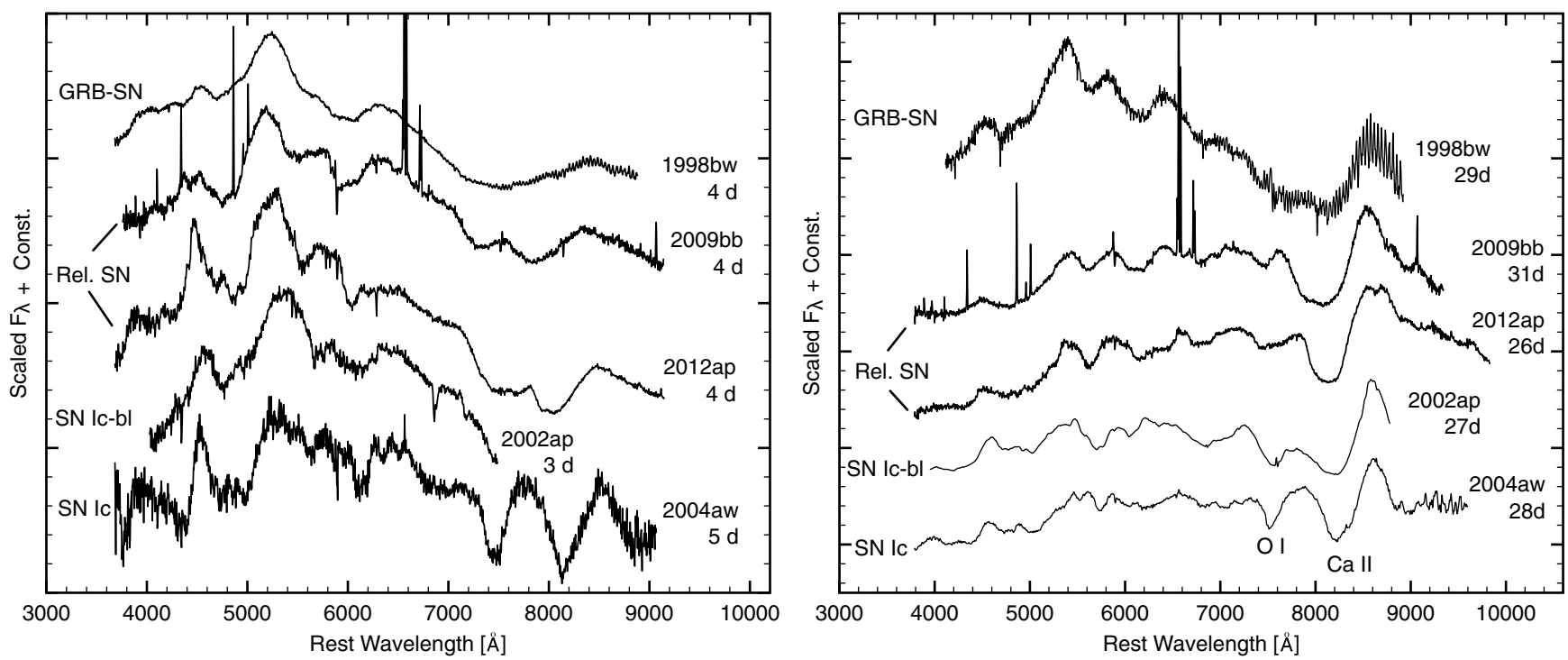

Figure 8. Left: optical spectrum of SN 2012ap around maximum light compared to that of various SN Ic and Ic-bl. Right: optical spectrum of SN 2012ap around one month post-maximum compared to that of various SN Ic and Ic-bl. Spectra have been downloaded from the SUSPECT and WISEREP databases and were originally published in the following papers: SN 1998bw (Patat et al. 2001), SN 2009bb (Pignata et al. 2011), SN 2002ap (Foley et al. 2003), and SN 2004aw (Taubenberger et al. 2006).

powered by gamma rays arising from the ${ }^{56} \mathrm{Co}$ decay, gamma rays from electron-positron annihilation, and the kinetic energy of the positrons (Sutherland \& Wheeler 1984; Cappellaro et al. 1997). We also assumed that the rise time was 13 days. Wheeler et al. (2014) present a detailed outline of all assumptions and caveats used in the Arnett (1982) model that we have adopted.

Using a conservative estimate of the photospheric velocity (PV) of $20,000 \mathrm{~km} \mathrm{~s}^{-1}$ (see Section 4.3), modeling of our data yields the following values for the explosion parameters: $M_{\mathrm{Ni}}=0.12 \pm 0.02 M_{\odot}, E_{k}=(0.9 \pm 0.3) \times 10^{52} \mathrm{erg}$, and $M_{\mathrm{ej}}=2.7 \pm 0.5 M_{\odot}$. The quoted uncertainties come from a range of optical opacities that were evaluated from $k_{\mathrm{opt}}=$ $0.05-0.1 \mathrm{~cm}^{2} \mathrm{~g}^{-1}$.

\subsection{Spectral Evolution}

The early optical spectra of SN 2012ap (Figure 3) exhibit broad features dominated by Fe II, Ca II, and O I with velocities starting at about $20,000 \mathrm{~km} \mathrm{~s}^{-1}$ as measured from our earliest observation on day -3.4 . These ions and velocities are not unlike those observed in many SN Ic-bl. In Figure 8, spectra of SN 2012ap observed near the time of maximum light and around day 30 are shown and compared to those of various SN Ic-bl. Also shown is the Type Ic SN 2004aw, which was interpreted as being transitional between SN Ic and SN Ic-bl (Taubenberger et al. 2006).

Around epochs near maximum light, the spectral features of SN 2012ap straddle those observed in the SN 1998bw and the SN 2004aw at the extremes. Specifically, the absorptions of SN 2012ap are not as broad as those observed in SN 1998bw, nor are they as narrow, numerous, or weak as those observed in SN 2004aw. By day 30, the spectral features show less diversity and the P-Cygni profile of the Ca II near-infrared triplet is the most conspicuous feature in all examples shown. Interestingly, OI $\lambda 7774$ is stronger in progression from SN 1998bw to SN 2004aw, which parallels the approximate order of decreasing kinetic energy.

\subsection{Spectral Features}

We utilized the fast and direct P-Cygni summation code SYN++ to assess the atomic makeup of spectral features for SN 2012ap and simultaneously extract projected Doppler velocities (see Thomas et al. 2011 for details of model parameters). Most ions are associated with an exponential line optical depth profile starting at the (assumed sharp) PV. Other species are then "detached" above the photosphere at high or very high velocities (HV, VHV) when necessary (see Branch et al. 2006 and Parrent et al. 2007). The excitation temperature, temp, has been fixed to $7000 \mathrm{~K}$, and we utilize the quadratic warping constant a2 (in addition to $\mathrm{a} 0$ and a1) in order to reduce the parameter space associated with needing an overly effective source of line-blanketing blueward of $5000 \AA$ for a given blackbody continuum level.

In Figure 9, we show a representative SYN++ best fit for a nearmaximum and post-maximum light spectrum of SN 2012ap. From our analysis, both observed spectra are primarily consistent with signatures of Ca II and Fe II between 19,000 and $14,000 \mathrm{~km} \mathrm{~s}^{-1}$. Fe I is not explicitly detected; however, its introduction provides the necessary enhanced line-blanketing between 4000 and $5000 \AA$ without conflicting elsewhere in the fit (e.g., as in the case of Co I). Contribution from Mg I cannot be ruled out. The small change in measured velocity implies a shallow change in the PV in the 23 days that separate the observations.

Near maximum light, a large absorption trough is observed at 7000-8500 A. We find fair agreement with multiple components of Ca II, including detached components of HV and VHV Ca II at 35,000 and $42,000 \mathrm{~km} \mathrm{~s}^{-1}$, respectively. For these inferred components of Ca-rich material, the fit is convincing so far as the observed absorption features are not largely consistent with photospheric O I and/or Mg II. Similar to previous studies of SN Ibc spectra, we find a degeneracy between PV He I and Na I for the absorption feature at $5560 \AA$ (see Valenti et al. 2011).

Our best fit for the absorption feature at $6050 \AA$ near maximum light is detached $\mathrm{H}$ i at $27,000 \mathrm{~km} \mathrm{~s}^{-1}$. As shown in Figure 10, use of PV Si II for the $6050 \AA$ feature produces 


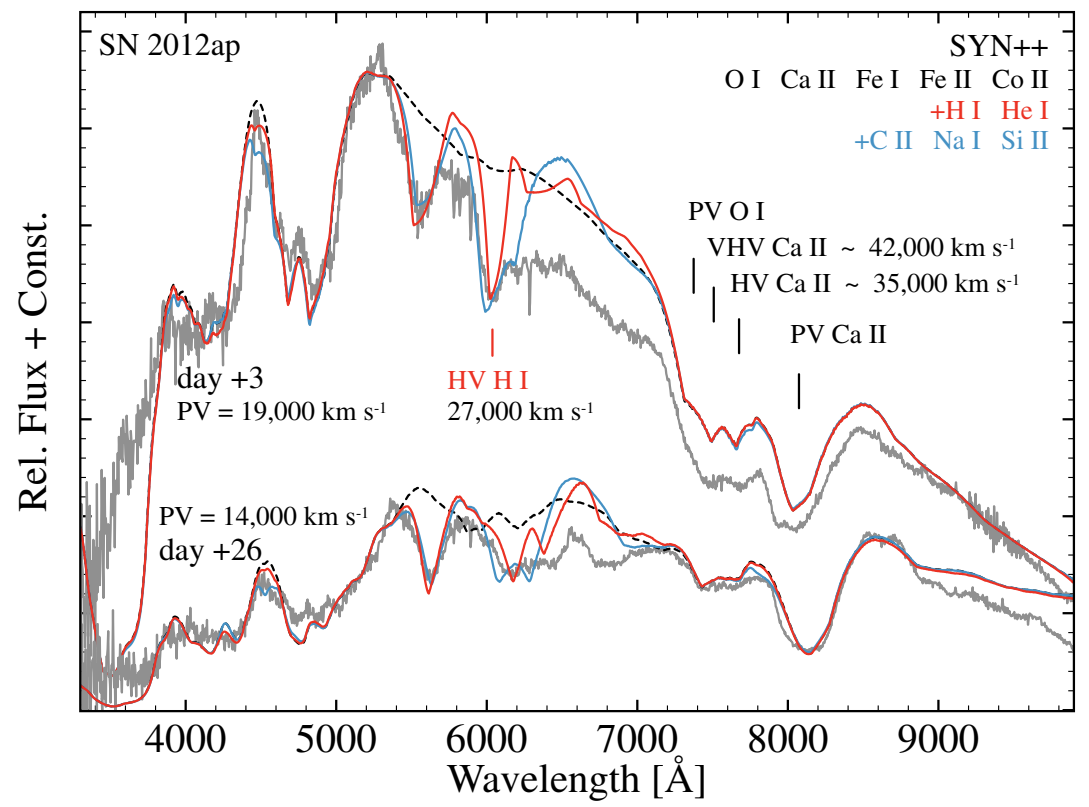

Figure 9. SYN++ synthetic spectrum comparisons to day +3 and day +26 observations of SN 2012ap (in gray). Ions considered for our fits are shown in the top right corner. The dashed black line shows our "base model" of assumed ions, while the red and blue lines represent the full fit with H I and He I, and C II, Na I, and Si II, respectively. All ions presented are at the labeled photospheric velocity (PV), unless stated otherwise.
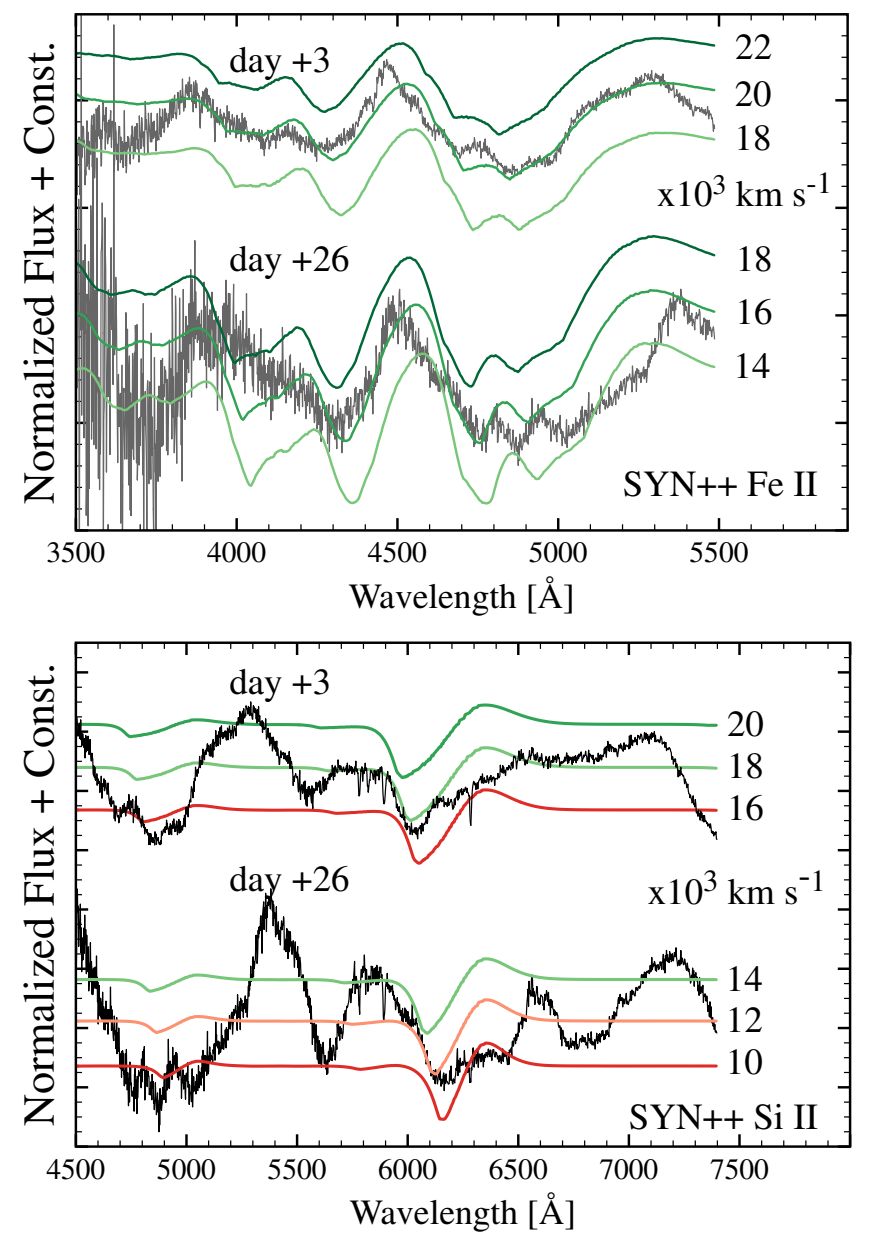

Figure 10. Individual ion fits for Fe II and Si II. For both the day +3 and day +26 spectrum, Fe II is most consistent with projected Doppler velocities of $20,000 \mathrm{~km} \mathrm{~s}^{-1}$ and $16,000 \mathrm{~km} \mathrm{~s}^{-1}$, respectively. The projected Doppler velocities for $\mathrm{Si}$ II at these same epochs is $\sim 2000-4000 \mathrm{~km} \mathrm{~s}^{-1}$ below those of Fe II. absorptions that are consistently too blue throughout postmaximum epochs. Resolving the fit in favor of Si II would require a lower PV, while detaching all other species. This is not a reasonable physical situation (Jeffery \& Branch 1990; Ketchum et al. 2008; James \& Baron 2010). Introduction of PV $\mathrm{Si}$ II improves the overall fit, but it is not a dominant contributor to any individual feature.

In order to test the consistency of our spectroscopic interpretations between adjacent wavelength regions, in Figure 11 we extend our best optical SYN++ fit out to near-infrared wavelengths using the Magellan/FIRE spectrum obtained on day +14 . We find consistency with the inference of He I at optical wavelengths and the large $1 \mu \mathrm{m}$ absorption trough, although the He I $\lambda 10830$ cannot fully account for the breadth of the $1 \mu \mathrm{m}$ feature. Other ions such as $\mathrm{Mg}$ II, S I, and C I are plausible contributors to the $1 \mu \mathrm{m}$ absorption, but each of these species can be immediately ruled out as solely responsible given respective conflicts at neighboring wavelengths. Reducing optical depths is not enough to sufficiently hide these conflicting absorption signatures without invoking non-local thermodynamic equilibrium (LTE) effects.

An additional test for helium is the presence of the He I 220587 line (Taubenberger et al. 2006). There is evidence of absorption in the $2 \mu \mathrm{m}$ region of the near-infrared spectrum of SN 2012ap, but the $\mathrm{S} / \mathrm{N}$ is poor and the absorption, if present, would be at a level where the relative flux ratio $R=\lambda 20587 /$ $\lambda 10830 \ll 1$. Models favor that if the He I $\lambda 10830$ line is present, then the He I $\lambda 20587$ line should also be visible at comparable strength $(R \approx 1$; Mazzali \& Lucy 1998).

However, the strength of the He I lines is expected to increase with time, as gamma rays penetrate further, since the excited levels from which He I lines form are populated almost exclusively by non-thermal processes (Mazzali \& Lucy 1998). Indeed, the strength of the He I $\lambda 20587$ line has been seen to increase over time (Marion et al. 2014). Since the only near-infrared spectrum of SN 2012ap has an epoch of 14 days after maximum, it is possible that the He I $\lambda 10830$ and $\lambda 20587$ lines have not fully 


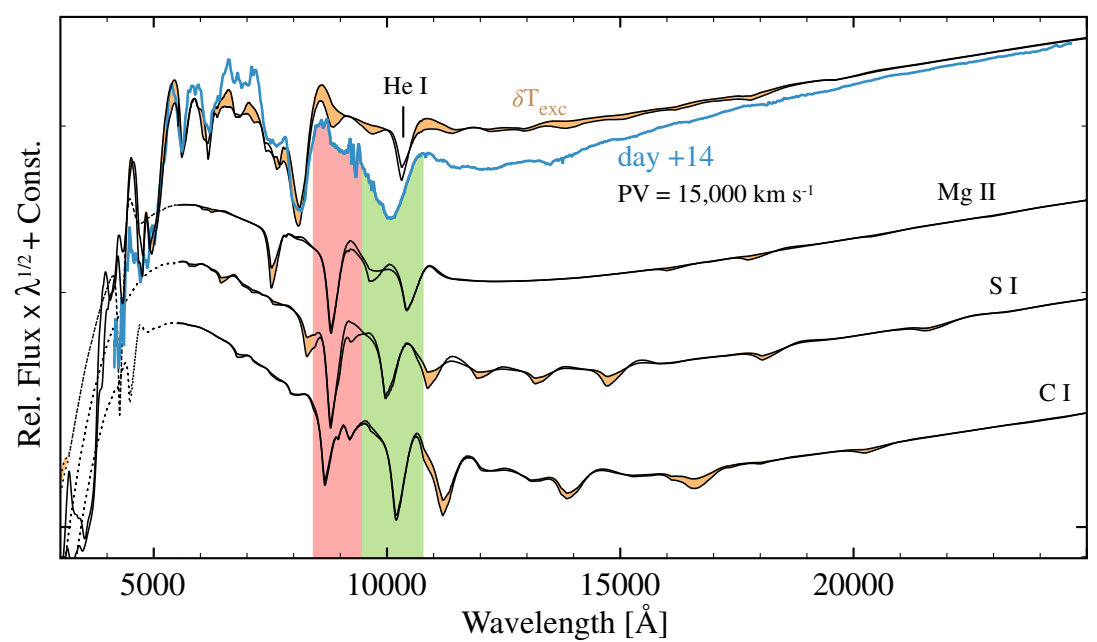

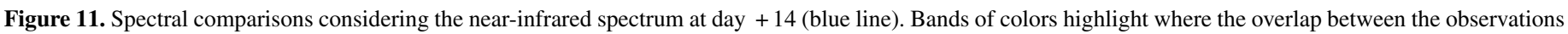

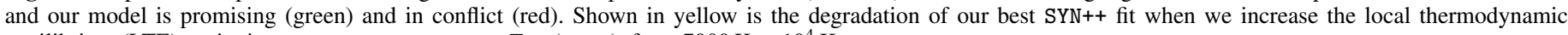
equilibrium (LTE) excitation temperature parameter, $T_{\text {exc }}$ (temp), from $7000 \mathrm{~K}$ to $10^{4} \mathrm{~K}$.

developed yet. The strength of the optical lines of He I should be proportional to those in the near-infrared.

We consider the detection of helium-rich ejecta as reasonably viable. That He I is unable to solely account for the $1 \mu \mathrm{m}$ absorption does not rule it out from contributing to this compound feature. It is reasonable to suspect that near-infrared wavelengths are sampling more layers than accounted for by the simplified LTE SYN++ model (Wheeler et al. 1998; Branch et al. 2002; Dessart et al. 2011). Furthermore, non-LTE calculations with the radiative transfer code PHOENIX (see, e.g., Hauschildt \& Baron 1999, 2014) have demonstrated that permitted lines of Fe II may contribute absorption features blueward of $1 \mu \mathrm{m}$ (Friesen et al. 2014).

\subsection{Analysis of Nebular Spectra}

Optical spectra of SN 2012ap during the nebular phase $(t>$ 200 days; Figure 5) exhibit conspicuous emissions associated with [O I] $\lambda \lambda 6300,6364,[\mathrm{Ca}$ II] $\lambda \lambda 7291,7324$, and the Ca II triplet around $8600 \AA$. These emissions originating from inner metal-rich ejecta heated by radioactive ${ }^{56} \mathrm{Co}$ are typical of SN Ibc at late stages (Filippenko et al. 1990; Mazzali et al. 2001; Matheson et al. 2001; Taubenberger et al. 2009; Modjaz et al. 2014). Other emission lines that are also often observed at these epochs are weakly detected, including $\mathrm{Mg} \mathrm{I}] \lambda 4571$, $\mathrm{Na}$ I D, and additional emission around $5200 \AA$ associated with blends of [Fe II].

Figure 12 shows a representative late-time spectrum of SN 2012ap compared to spectra of other SN Ic-bl. The MgI] $\lambda 4571$ line is particularly weak in the day 272 spectrum, although our observations do suffer from reduced sensitivity in this wavelength region. The relative line flux ratio of [Ca II]/ $\left[\mathrm{O}_{\mathrm{I}}\right] \approx 1.2$ is large in SN 2012ap compared to those observed in SN 1998bw and SN 2002ap where it is $\approx 0.5$. SN 2009bb and SN 2007ru also show $[\mathrm{CaII}] /[\mathrm{OI}]>1$. This line flux ratio has been suggested to be a useful indicator of progenitor core mass, with larger $[\mathrm{Ca} I \mathrm{II}] /\left[\mathrm{O}_{\mathrm{I}}\right]$ ratios indicative of a less massive helium core at the time of explosion (Fransson \& Chevalier 1989).

Emission-line velocity plots comparing the lines in SN 2012ap to those of other SN Ic-bl are shown in Figure 13. The observed [O I] and [Ca II] emissions exhibit line velocities of $\sim 5500 \mathrm{~km} \mathrm{~s}^{-1}$ that are not unlike those observed in other
SN Ic-bl (Maurer et al. 2010). The [Ca II] $\lambda \lambda 7291,7324$ emission is noticeably broad, and its blueshifted velocities appear to be greater than those of oxygen. However, emission blueward of $7306 \AA$ (which we assume to be the center of the distribution) has likely contribution from the [Fe II] $\lambda 7155$ and $\lambda 7172$ lines. The single peak of the entire [Ca II] distribution is blueshifted by $1700 \mathrm{~km} \mathrm{~s}^{-1}$ and has a sharp dropoff on the redshifted side.

The [O I] emission line profile appears to be double-peaked. The blueshifted and strongest peak in the distribution is centered near $-1700 \mathrm{~km} \mathrm{~s}^{-1}$, which is the same velocity as the peak observed in the [Ca II] distribution. The minimum between the two peaks is near $6300 \AA\left(\approx 0 \mathrm{~km} \mathrm{~s}^{-1}\right)$. Between days 218 and 272, emission redward of $6300 \AA$ increases in strength. This evolution, illustrated in Figure 14, is discussed further in Section 5.1.

We modeled the nebular spectrum using our SN nebular spectrum code, assuming that the late-time emission is tied to the deposition of gamma rays and positrons from ${ }^{56} \mathrm{Co}$ decay. Given an ejected mass, a characteristic boundary velocity (which corresponds to the half width at half-maximum intensity of the emission lines), and a composition, the code computes gammaray deposition, follows the diffusions of the gamma rays and the positrons with a Monte Carlo scheme, and computes the heating of the gas. The state of the gas is then computed in non-LTE, balancing heating and cooling via line emission. The code has been used for a number of SN Ibc (e.g., Mazzali et al. 2001, 2007, 2010) and is the latest version described in some detail by Mazzali et al. (2011).

The synthetic spectrum produced by our model for the day 218 optical spectrum is shown in Figure 15. For material inside $5500 \mathrm{~km} \mathrm{~s}^{-1}$ we derive a nickel mass of $M_{\mathrm{Ni}}=0.20 \pm 0.05 M_{\odot}$. This estimate of $M_{\mathrm{Ni}}$ is larger than that derived from modeling of the bolometric light curve in Section $4.1\left(0.12 \pm 0.02 M_{\odot}\right)$, but not grossly inconsistent. Additionally, we estimate the oxygen mass to be $M_{\text {oxygen }} \approx 0.5 M_{\odot}$ and the total ejecta mass to be $M_{\mathrm{ej}} \approx 0.8 M_{\odot}$. The value of $M_{\mathrm{ej}}$ calculated by our models of the nebular spectra is less than that derived in Section 4.1 $\left(\sim 2.7 M_{\odot}\right)$. Some of the discrepancy is because this value is only for mass inside $5500 \mathrm{~km} \mathrm{~s}^{-1}$. Also, as we show in Section 5.1, there is a possibility of internal absorption. If the emission lines are not optically thin, then the masses derived from them will be underestimated. 


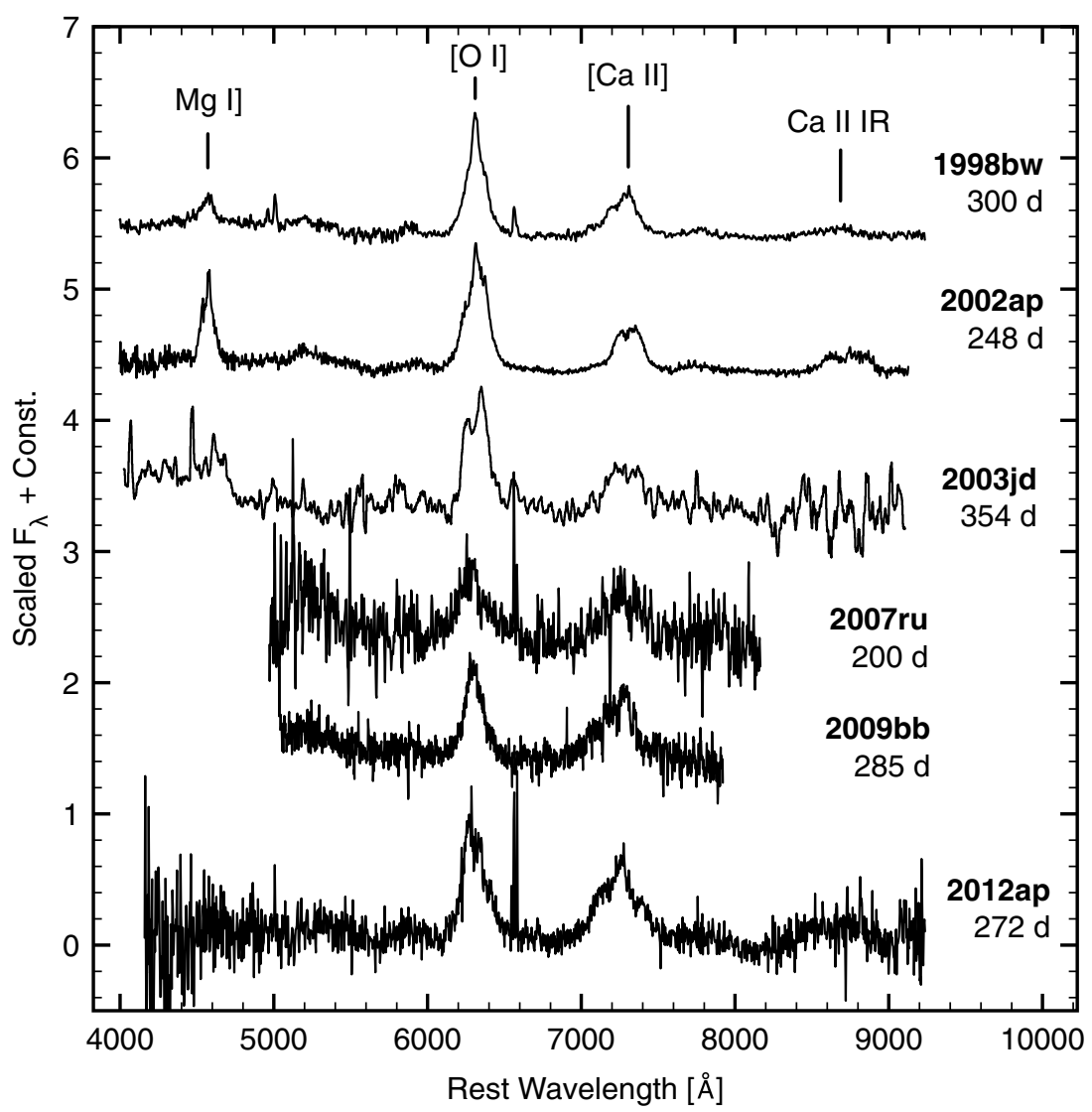

Figure 12. Nebular spectrum of SN 2012ap compared to that of other SN Ic-bl. Spectra have been downloaded from the SUSPECT and WISEREP databases and were originally published in the following papers: SN 1998bw (Patat et al. 2001), SN 2002ap (Taubenberger et al. 2009), SN 2003jd (Valenti et al. 2008b), SN 2007ru (Sahu et al. 2009), and SN 2009bb (Pignata et al. 2011). Emission labeled as [Ca II] has potential contributions from [Fe II] $\lambda 7155$ and $\lambda 7172$, and Ca II IR has potential contributions from $[\mathrm{Fe}$ II] $\lambda 8617$ and $[\mathrm{C} \mathrm{I}] \lambda 8727$.

\section{DISCUSSION}

Our UV, optical, and near-infrared observations of SN 2012ap show it to be a member of the energetic SN Ic-bl class with explosion properties that fall in between normal SN Ibc events and SNe-GRBs. SN 2012ap follows SN 2009bb as the second example of a SNIc-bl associated with ejecta moving at relativistic velocities $(v \gtrsim 0.6 c)$ but not associated with a GRB detection. In the case of SN $2009 \mathrm{bb}$, its relativistic ejecta continued to be in nearly free expansion for $\sim 1 \mathrm{yr}$, which is unlike GRBs. SN 2012ap's relativistic ejecta, however, did slow down on timescales similar to those of GRBs (Chakraborti et al. 2014).

A very small percentage of SN explosions $(<1 \%)$ harbor a central engine capable of powering an ultra-relativistic jet detected as a GRB (Soderberg et al. 2006a). Thus, it is of interest to compare and contrast the properties of SN 2009bb and SN 2012ap to those of other SN Ibc and GRB-SN. Below we discuss some of the more interesting observational properties of these relativistic SNe without a GRB detection uncovered in our analysis, and discuss their relevance to potential progenitor systems and explosion mechanisms.

\subsection{Hydrogen and Helium in SN Ic-bl}

Our multi-epoch SYN++ fits of the optical and near-infrared spectra of SN 2012ap strongly favor the identification of helium in the ejecta. Detection begins from the first observation on day -3.4 and PVs of $20,000 \mathrm{~km} \mathrm{~s}^{-1}$ are measured. In addition, hydrogen is likely to be present and detached above the photosphere traveling at $\sim 27,000 \mathrm{~km} \mathrm{~s}^{-1}$. Helium was also detected in pre-maximum light spectra of SN 2009bb (Pignata et al. 2011), though in that case it was not as conspicuous, and hydrogen was not reported.

It is not unusual for helium to be detected in the spectra of SN Ic and SN Ic-bl. Observations of sufficient quality and temporal coverage in combination with spectral modeling have shown that absorption features in a variety of SN Ibc spectra can sometimes be best understood as arising from helium (Filippenko 1988, 1992; Filippenko et al. 1995; Clocchiatti et al. 1996; Patat et al. 2001; Mazzali et al. 2002; Elmhamdi et al. 2006; Branch et al. 2006; Bufano et al. 2012). In some of these instances, hydrogen traveling at velocities above the photosphere has also been identified as a possible constituent of the ejecta.

However, the extent of $\mathrm{H}$ and $\mathrm{He}$ in SN Ibc, and especially SN Ic-bl, remains debated (Matheson et al. 2001; Branch et al. 2002, 2006; Hachinger et al. 2012; Milisavljevic et al. 2013; Modjaz et al. 2014; Piro \& Morozova 2014). It is particularly difficult to derive masses of He because of the high excitation potentials of He that exceed the energy of thermal photons and electrons, and require detailed non-LTE spectral modeling. The presence or lack of He I lines in SN Ibc may indicate a genuine helium deficiency, or, alternatively, the result of inadequate excitation (Harkness et al. 1987; Dessart et al. 2011; Li et al. 2012). Only a small amount of helium $\left(\lesssim 0.1 M_{\odot}\right)$ needs to be present to be observable (Hachinger et al. 2012), but asymmetric mixing or weak mixing may prevent sizable amounts of $\mathrm{He}$ from being excited by Co (Dessart et al. 2012). With time, 


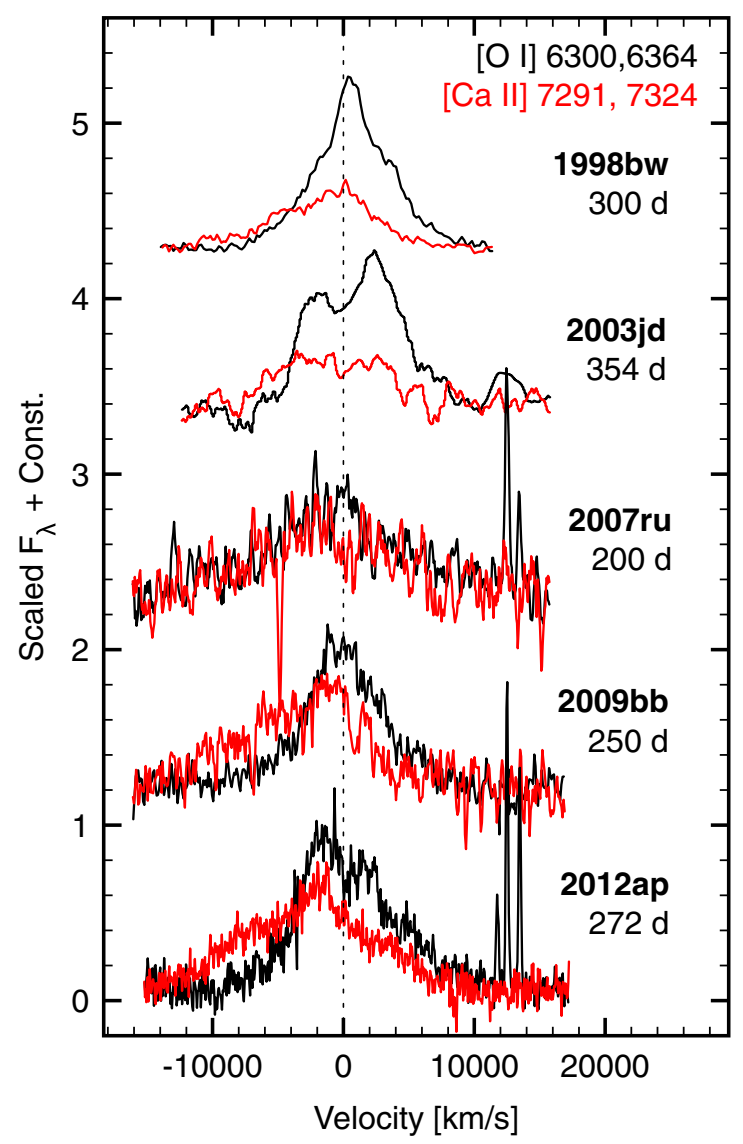

Figure 13. Emission-line profiles of [OI] $\lambda \lambda 6300,6364$ and [Ca II] $\lambda \lambda 7291$, 7324 observed in SN 2012ap and other broad-lined Type Ic supernovae from Figure 12. Velocities for [O I] are with respect to $6300 \AA$, and velocities for [Ca II] are with respect to $7306 \AA$. Flux values for [O I] are normalized to one and the relative flux between $[\mathrm{OI}] /[\mathrm{Ca}$ II] has been maintained. [Ca II] profiles are contaminated to varying degrees by the $[\mathrm{Fe} \mathrm{I}] \lambda 7155$ and $\lambda 7172$ lines.

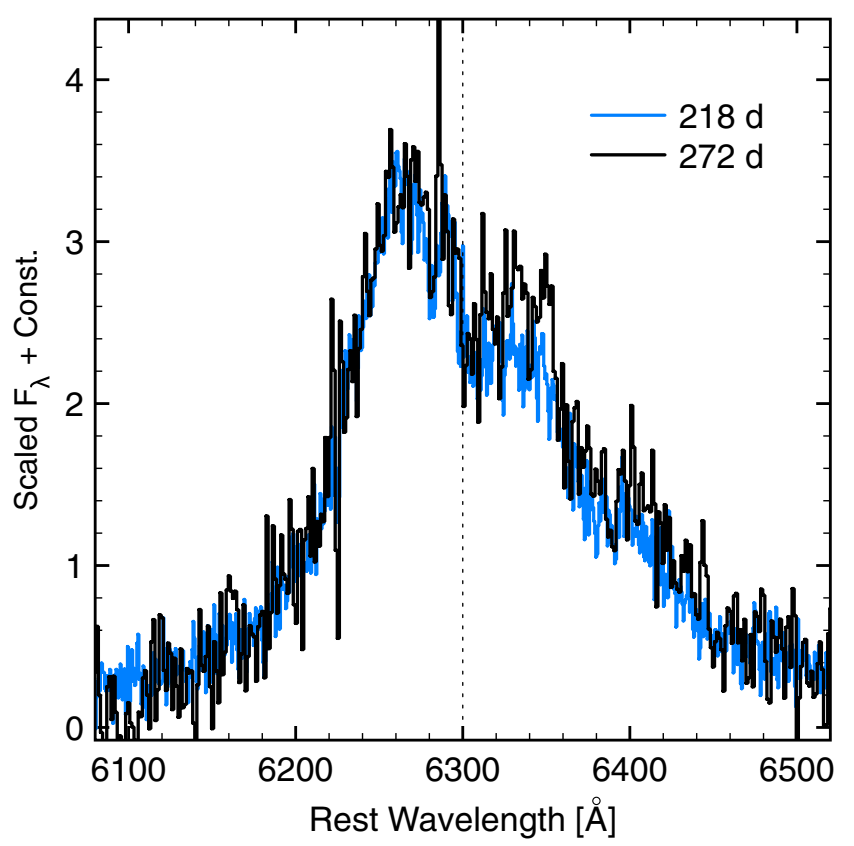

Figure 14. Late-time emission of [O I] $\lambda \lambda 6300,6364$ in SN 2012ap between day 218 and 272. Emission redward of $6300 \AA$ increases with time.

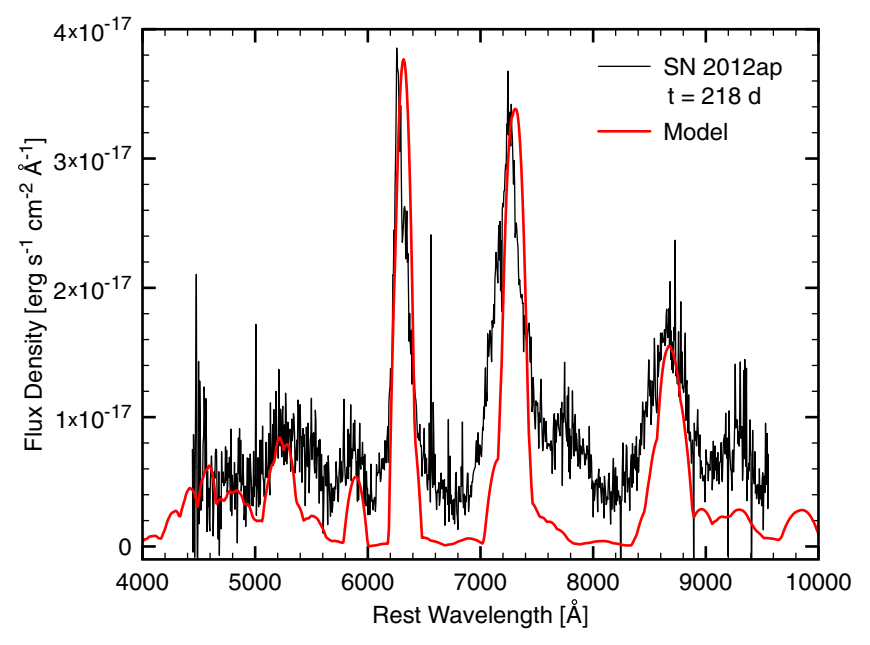

Figure 15. Late-time optical spectrum of SN 2012ap obtained 2012 September $23(t=218$ days; black) and our model fit (red).

SN expansion will thin the ejecta and should eventually expose any He-rich material to radioactive decay (Swartz et al. 1993).

Determining the amount of $\mathrm{H}$ and $\mathrm{He}$ in $\mathrm{SN}$ ejecta is an important constraint on the evolutionary state of the progenitor star at the time of explosion. There is ample reason to believe that sizable quantities of these elements should remain, as no completely hydrogen-free low-mass helium stars are known (Eldridge et al. 2013), and stellar models have difficulties explaining strong loss of He (Georgy et al. 2009; Yoon et al. 2010). Recent stellar evolution calculations predict that the mass of He can reach upward of $0.5 M_{\odot}$ in SN Ibc progenitors.

\subsection{Asymmetric [O I] Emission}

Conspicuous asymmetry in the [O I] $\lambda \lambda 6300,6364$ profile of SN 2012ap is suggestive of two peaks (Figure 14). Examinations of late-time spectra of many SN Ibc $(\gtrsim 40)$ have demonstrated that double-peaked line profiles in [OI] emission seem to be a relatively common phenomenon occurring in 30\%-40\% of objects (Maeda et al. 2008; Modjaz et al. 2008; Taubenberger et al. 2009; Milisavljevic et al. 2010). Such double-peaked emission-line profiles deviate from the single-peaked profile expected from a spherically symmetric source, and this has been interpreted as evidence for aspheric debris having a toroidal or disk-like geometry (Maeda et al. 2002). Mazzali et al. (2005) observed a double-peaked [O I] profile in the SN Ic-bl 2003jd and interpreted it as emission originating from a torus of O-rich debris perpendicular to a high-velocity jet in a GRB model. In this framework, nondetections of GRBs from SN Ic-bl are the consequence of viewing jet-driven explosions along the equatorial expansion plane perpendicular to the jet axis.

The double-peaked [O I] profile observed in SN 2012ap could be produced by a toroidal ejecta geometry similar to the model of Mazzali et al. (2005). If so, the distribution of O-rich material in SN 2012ap was not as aspheric as in SN 2003jd because the peaks observed in SN2012ap are neither as pronounced nor as widely separated (see Figure 13). Notably, SN 2009bb does not exhibit considerable asymmetry in its [O I] distribution, although Pignata et al. (2011) report that the signature of aspheric explosion dynamics might have been seen in earlier nebular data.

Alternatively, the observed [OI] profile of SN 2012ap could be the result of absorption in the SN interior. The evolution in the 
relative strength of the two peaks of [O I] emission is consistent with this scenario. The two peaks are divided by a steep dropoff in emission near zero velocity, and the strength of emission redward of zero velocity increases over time (Figure 14). The fact that [CaII] is asymmetric and preferentially blueshifted with a distribution similar to that observed in [O I] is also consistent with a significant portion of interior ejecta being opaque (Figure 13).

Possible contributors to this opacity are high densities or dust in the ejecta. Optically thick inner ejecta could prevent light from the rear side of the SN from penetrating, creating a flux deficit in the redshifted part of emission lines (Taubenberger et al. 2009; Milisavljevic et al. 2010), as observed in the late-time emission profiles of SN 2012ap (Figure 13). Internal absorption associated with high densities is generally not anticipated at these late epochs $(t \gtrsim 300$ days $)$ because SN expansion should lead to low densities with associated low optical opacities (Maeda et al. 2008). However, deviations from spherical symmetry and clumping make it possible for portions of the ejecta to remain optically thick (Spyromilio et al. 1990; Li \& McCray 1992; Chugai 1994).

Dust formation is not directly supported by our observations. There is no change in the blueshifted position of the peaks toward smaller wavelengths (Lucy et al. 1989), and no latetime infrared observations are available to detect potential flux excess at wavelengths that would be associated with heated dust emission (see, e.g., Dwek et al. 1983; Kozasa et al. 1991; Gerardy et al. 2002; Fox et al. 2009).

\subsection{Host Environment and Progenitor System}

Several properties of SN 2012ap and SN 2009bb distinguish them from most SN Ic-bl and may have implications for the progenitor systems of relativistic SNe lacking a GRB detection. One such property is their host environment metallicities. The oxygen abundance of the explosion site of SN 2012ap using the N2 scale of Pettini \& Pagel (2004) is $\log (\mathrm{O} / \mathrm{H})+12=8.79$ (M14), which is slightly above $\operatorname{solar}\left(Z_{\odot} \equiv \log (\mathrm{O} / \mathrm{H})_{\odot}+12=\right.$ 8.69; Asplund et al. 2005). The host metallicity of SN 2009bb was estimated to be $1.7-3.5 Z_{\odot}$ (Levesque et al. 2010). These metallicities are at the high end of environments of the majority of SN Ic-bl discovered by targeted surveys, and substantially higher than those of $\mathrm{SNe}$ discovered in untargeted surveys where the mean metallicity is $\sim Z_{\odot} / 2$ (Anderson et al. 2010; Modjaz et al. 2011; Kelly \& Kirshner 2012; Sanders et al. 2012a). SN 2009bb and SN 2012ap were both discovered by targeted surveys.

It is worth noting that both $\mathrm{SNe}$ were located in the outer regions of their host galaxies along the edges of spiral arms. This peripheral location is unlike the majority of SN Ic that are much more likely to occur in the brightest regions of their host galaxies (Kelly et al. 2008, 2014). The outer location also stands in contrast with the fact that the environmental metallicity of spiral galaxies typically decreases as a function of radial distance from the core (Zaritsky et al. 1994), although this trend is known to have important exceptions (Zurita \& Bresolin 2012).

Wolf-Rayet (WR) stars have long been a suspected progenitor system of SN Ibc (Gaskell et al. 1986; Gal-Yam et al. 2014, and references therein). However, it is now thought that WR stars are unlikely to be the dominant progenitor channel. Luminosity limits derived from nondetections of progenitor stars in highresolution pre-explosion images (Van Dyk et al. 2003; Smartt 2009), have ruled out a large population of WR stars as originators of SN Ibc. Instead, relatively low-mass helium stars in binary systems may represent a large fraction of SN Ibc (Eldridge et al. 2008, 2013; Smith et al. 2011).

In the case of SN 2012ap, there is in fact circumstantial evidence in support of a WR progenitor system association. M14 report that the unusually strong DIBs in the optical spectra exhibit changes in EW over short ( $\lesssim 30$ days) timescales in a manner consistent with the SN interacting with a nearby source of the DIBs. The only other reported cases of time-varying DIB features have been in families of WR and luminous blue variable (LBV) stars (Le Bertre \& Lequeux 1993; Heydari-Malayeri et al. 1993).

Interestingly, SN 2009bb was also found to be associated with strong DIB absorptions in its moderately extinguished optical spectra, as was SN 2008D, which exhibited broad-lined features similar to SN 2012ap before maximum light but then evolved into a SN Ib. Although chance alignments between DIB carrierrich molecular clouds and these $\mathrm{SNe}$ are possible, it may be that the SN progenitor systems are related to the sources of the DIBs, and that the source could be related to mass loss from the progenitor star. LBV and WR stars exhibit varying degrees of asymmetric mass loss (Nota et al. 1995); thus, an observer's line of sight with respect to a circumstellar disk could be an important factor in explaining why strong DIB detections are rare.

\section{CONCLUSIONS}

We have presented ultraviolet, optical, and near-infrared observations of the broad-lined Type Ic SN 2012ap, which is the second known example of a SN from a stripped-envelope progenitor capable of accelerating a non-negligible portion of its ejecta to relativistic velocities $(v>0.6 c)$ but not associated with a GRB detection. The only known counterpart is SN 2009bb.

Notable properties of these two relativistic SN Ic-bl may reflect key aspects of their progenitor systems and explosion dynamics. In particular, both SN 2012ap and SN 2009bb share the following characteristics.

1. Strong radio emission consistent with ejecta being accelerated to relativistic velocities but no GRB counterpart.

2. Weak X-ray emission at late times ( $t>10$ days $)$.

3 . Evidence for helium in early-time optical spectra with photospheric velocities of $\gtrsim 20,000 \mathrm{~km} \mathrm{~s}^{-1}$.

4. Relatively large emission-line flux ratio of $[\mathrm{Ca}$ II $] /\left[\mathrm{O}_{\mathrm{I}}\right]>1$ in nebular spectra.

5. High levels of internal host extinction $(E(B-V)>$ $0.4 \mathrm{mag}$ ).

6. Environments of solar to super-solar metallicity, and locations along the outer spiral arms of their host galaxies.

Margutti et al. (2014) propose that SN 2009bb and SN 2012ap represent the weakest of central-engine-driven explosions, and conclude that these events lack an associated GRB detection because engine activity stops before the jet is able to pierce through the stellar envelope. Though "choked," the jet is still able to accelerate a small fraction of ejecta to relativistic velocities. Engines of short durations or progenitors of large stellar envelopes may inhibit the jet from completely piercing the surface of the star.

Indeed, SN 2009bb and SN 2012ap may be among the weakest explosions for which we are able to detect the presence of jet activity. The continuum of explosions extending from GRBSN to more ordinary SN Ibc suggests that a wider variety of jet activity may potentially be occurring at energies that are observationally "hidden." Detection at weaker scales is challenging 
since such explosions do not produce electromagnetic signatures as easily recognizable as GRBs. SNe of this variety can be dynamically indistinguishable from ordinary core-collapse SN (Tan et al. 2001; Matzner 2003; Lazzati et al. 2012), and/or their high-energy emissions may be below the threshold of the current generation of gamma-ray instruments (Pignata et al. 2011).

Some hints in support of jets at smaller energy scales come from SN remnants. Cassiopeia A, known to be the result of an asymmetric Type IIb explosion (Krause et al. 2008; Rest et al. 2011), exhibits exceptionally high velocity Si- and S-rich material in a jet/counter-jet arrangement (Fesen 2001; Hines et al. 2004; Hwang et al. 2004; Fesen et al. 2006). The known extent of this jet region contains fragmented knots of debris traveling $\sim 15,000 \mathrm{~km} \mathrm{~s}^{-1}$, which is three times the velocity of the bulk of the $\mathrm{O}$ - and S-rich main shell. Though the large opening half-angle of this high-velocity ejecta is inconsistent with a highly collimated flow (Milisavljevic \& Fesen 2013), some jet-like mechanism carved a path allowing interior material from the $\mathrm{Si}-\mathrm{S}-\mathrm{Ar}-\mathrm{Ca}$ region near the core out past the mantle and $\mathrm{H}$ - and He-rich photosphere. This process, potentially related to a protoneutron star wind that follows the SN outburst (Burrows 2005), would be observationally indistinguishable from non-jet explosion models at extragalactic distances.

Rotation is believed to be a key variable driving the outcome of these explosions. If the jet is associated with the protoneutron star or magnetar wind that follows the SN, rapid rotation will naturally amplify magnetic fields and make magnetohydrodynamic power influential (Akiyama et al. 2003). GRBs may only come from the most rapidly rotating and most massive stars (Woosley \& Bloom 2006; Burrows et al. 2007).

Progenitor composition and structure is another important consideration in SN explosions (Arnett \& Meakin 2011; Ugliano et al. 2012). He and/or H layers that can vary in thickness along different viewing angles (Maund et al. 2009), have the potential to quench relativistic jets (e.g., SN 2008D; Mazzali et al. 2008b), and can lead to expansion asymmetries that can be potentially detected by spectropolarimetry (Maund et al. 2007; Wang \& Wheeler 2008; Tanaka et al. 2008, 2012). The inferred presence of helium in the optical spectra of SN 2012ap and SN 2009bb is consistent with the quenched jet scenario.

With only two events identified so far, it is possible that the exceptional properties of SN 2012ap and 2009bb discussed here are biased by transient surveys targeting metal-rich systems. Additional examples of relativistic SN Ic-bl are needed to test whether the properties maintain and to further understand these events in the entire context of SN Ibc. Given their complex origins, e.g., line-of-sight influences of potentially asymmetric explosions inside progenitors of varying structure and compositions, varying core-rotation speeds, and asymmetric circumburst mediums of differing metallicities, real progress requires an in-depth and multi-wavelength (radio through gamma-rays) approach studying a large sample $(N>30)$ of local SN Ic-bl.

We thank an anonymous referee for helpful suggestions. Many of the observations reported in this paper were obtained with the Southern African Large Telescope. Additional data presented herein were obtained at the W. M. Keck Observatory, which is operated as a scientific partnership among the California Institute of Technology, the University of California, and NASA; the observatory was made possible by the generous financial support of the W. M. Keck Foundation. Some observations also came from the MMT Observatory, a joint facility of the Smithsonian Institution and the University of Arizona, as well as the $6.5 \mathrm{~m}$ Magellan Telescopes located at Las Campanas Observatory, Chile. Support was provided by the David and Lucile Packard Foundation Fellowship for Science and Engineering awarded to A.M.S. J.M.S. is supported by an NSF Astronomy and Astrophysics Postdoctoral Fellowship under award AST-1302771. T.E.P. thanks the National Research Foundation of South Africa. R.P.K. and J.C.W. are grateful for NSF grants AST-1211196 and AST-1109801, respectively. A.V.F. and S.B.C. acknowledge generous support from Gary and Cynthia Bengier, the Richard and Rhoda Goldman Fund, the Christopher R. Redlich Fund, the TABASGO Foundation, and NSF grant AST-1211916. K.M. acknowledges financial support by Grant-in-Aid for Scientific Research for Young Scientists (23740141, 26800100). The work by K.M. is partly supported by World Premier International Research Center Initiative (WPI Initiative), MEXT, Japan. J.V. is supported by Hungarian OTKA grant NN-107637. M.D.S. and E.Y.H. gratefully acknowledge generous support provided by the Danish Agency for Science and Technology and Innovation realized through a Sapere Aude Level 2 grant. E.Y.H. also acknowledges support from NSF grant AST-1008343. D. Sahu and G. Pignata kindly provided archival spectra of SN 2007ru and SN2009bb, respectively. This paper made extensive use of the SUSPECT database (www.nhn.ou.edu/ suspect/) and the Weizmann interactive supernova data repository (www.weizmann.ac.il/astrophysics/wiserep). This work was supported in part by NSF grant No. PHYS-1066293 and the hospitality of the Aspen Center for Physics.

\section{REFERENCES}

Akiyama, S., Wheeler, J. C., Meier, D. L., \& Lichtenstadt, I. 2003, ApJ, 584,954

Anderson, J. P., Covarrubias, R. A., James, P. A., Hamuy, M., \& Habergham, S. M. 2010, MNRAS, 407, 2660

Arnett, W. D. 1982, ApJ, 253, 785

Arnett, W. D., \& Meakin, C. 2011, ApJ, 733, 78

Asplund, M., Grevesse, N., \& Sauval, A. J. 2005, in ASP Conf. Ser. 336, Cosmic Abundances as Records of Stellar Evolution and Nucleosynthesis, ed. T. G. Barnes, III \& F. N. Bash (San Francisco, CA: ASP), 25

Ben-Ami, S., Gal-Yam, A., Filippenko, A. V., et al. 2012, ApJL, 760, L33

Berger, E., Chornock, R., Holmes, T. R., et al. 2011, ApJ, 743, 204

Berger, E., Kulkarni, S. R., \& Chevalier, R. A. 2002, ApJL, 577, L5

Bersten, M. C., Benvenuto, O. G., Folatelli, G., et al. 2014, AJ, 148, 68

Bianco, F. B., Modjaz, M., Hicken, M., et al. 2014, ApJS, 213, 19

Bigelow, B. C., Dressler, A. M., Shectman, S. A., \& Epps, H. W. 1998, Proc. SPIE, 3355, 225

Branch, D., Benetti, S., Kasen, D., et al. 2002, ApJ, 566, 1005

Branch, D., Jeffery, D. J., Young, T. R., \& Baron, E. 2006, PASP, 118, 791

Breeveld, A. A., Landsman, W., Holland, S. T., et al. 2011, in AIP Conf. Proc. 1358, Gamma Ray Bursts 2010, ed. J. E. McEnery, J. L. Racusin, \& N. Gehrels (Melville, NY: AIP), 373

Brown, P. J., Holland, S. T., Immler, S., et al. 2009, AJ, 137, 4517

Bufano, F., Pian, E., Sollerman, J., et al. 2012, ApJ, 753, 67

Burgh, E. B., Nordsieck, K. H., Kobulnicky, H. A., et al. 2003, Proc. SPIE, 4841,1463

Burrows, A. 2005, in ASP Conf. Ser. 342, 1604-2004: Supernovae as Cosmological Lighthouses, ed. M. Turatto, S. Benetti, L. Zampieri, \& W. Shea (San Francisco, CA: ASP), 184

Burrows, A., Dessart, L., Livne, E., Ott, C. D., \& Murphy, J. 2007, ApJ, 664,416

Cao, Y., Kasliwal, M. M., Arcavi, I., et al. 2013, ApJL, 775, L7

Cappellaro, E., Mazzali, P. A., Benetti, S., et al. 1997, A\&A, 328, 203

Cardelli, J. A., Clayton, G. C., \& Mathis, J. S. 1989, ApJ, 345, 245

Chakraborti, S., \& Ray, A. 2011, ApJ, 729, 57

Chakraborti, S., Soderberg, A., Chomiuk, L., et al. 2014, arXiv:1402.6336

Chornock, R., Berger, E., Levesque, E. M., et al. 2010, arXiv:1004.2262

Chugai, N. N. 1994, ApJL, 428, L17 
Clocchiatti, A., Suntzeff, N. B., Covarrubias, R., \& Candia, P. 2011, AJ, 141,163

Clocchiatti, A., Wheeler, J. C., Brotherton, M. S., et al. 1996, ApJ, 462, 462

Clocchiatti, A., Wheeler, J. C., Phillips, M. M., et al. 1997, ApJ, 483, 675

Corsi, A., Ofek, E. O., Frail, D. A., et al. 2011, ApJ, 741, 76

Crawford, S. M., Still, M., Schellart, P., et al. 2010, Proc. SPIE, 7737, 773725

Dessart, L., Hillier, D. J., Li, C., \& Woosley, S. 2012, MNRAS, 424, 2139

Dessart, L., Hillier, D. J., Livne, E., et al. 2011, MNRAS, 414, 2985

Drout, M. R., Soderberg, A. M., Gal-Yam, A., et al. 2011, ApJ, 741, 97

Dwek, E., A'Hearn, M. F., Becklin, E. E., et al. 1983, ApJ, 274, 168

Eldridge, J. J., Fraser, M., Maund, J. R., \& Smartt, S. J. 2014, arXiv:1408.4142

Eldridge, J. J., Fraser, M., Smartt, S. J., Maund, J. R., \& Crockett, R. M. 2013, MNRAS, 436, 774

Eldridge, J. J., Izzard, R. G., \& Tout, C. A. 2008, MNRAS, 384, 1109

Elmhamdi, A., Danziger, I. J., Branch, D., et al. 2006, A\&A, 450, 305

Faber, S. M., Phillips, A. C., Kibrick, R. I., et al. 2003, Proc. SPIE, 4841, 1657

Fesen, R. A. 2001, ApJS, 133, 161

Fesen, R. A., Hammell, M. C., Morse, J., et al. 2006, ApJ, 645, 283

Filippenko, A. V. 1988, AJ, 96, 1941

Filippenko, A. V. 1992, ApJL, 384, L37

Filippenko, A. V. 1997, ARA\&A, 35, 309

Filippenko, A. V., Barth, A. J., Matheson, T., et al. 1995, ApJL, 450, L11

Filippenko, A. V., Li, W. D., Treffers, R. R., \& Modjaz, M. 2001, in ASP Conf

Ser. 246, IAU Colloq. 183, Small Telescope Astronomy on Global Scales, ed. B. Paczynski, W.-P. Chen, \& C. Lemme (San Francisco, CA: ASP), 121

Filippenko, A. V., Porter, A. C., \& Sargent, W. L. W. 1990, AJ, 100, 1575

Foley, R. J., Papenkova, M. S., Swift, B. J., et al. 2003, PASP, 115, 1220

Fox, O., Skrutskie, M. F., Chevalier, R. A., et al. 2009, ApJ, 691, 650

Fransson, C., \& Chevalier, R. A. 1989, ApJ, 343, 323

Fremling, C., Sollerman, J., Taddia, F., et al. 2014, A\&A, 565, A114

Friedman, S. D., York, D. G., McCall, B. J., et al. 2011, ApJ, 727, 33

Friesen, B., Baron, E., Wisniewski, J. P., et al. 2014, ApJ, 792, 120

Galama, T. J., Vreeswijk, P. M., van Paradijs, J., et al. 1998, Natur, 395, 670

Gal-Yam, A., Arcavi, I., Ofek, E. O., et al. 2014, Natur, 509, 471

Gaskell, C. M., Cappellaro, E., Dinerstein, H. L., et al. 1986, ApJL, 306, L77

Gehrels, N., Chincarini, G., Giommi, P., et al. 2004, ApJ, 611, 1005

Georgy, C., Meynet, G., Walder, R., Folini, D., \& Maeder, A. 2009, A\&A, 502,611

Gerardy, C. L., Fesen, R. A., Nomoto, K., et al. 2002, PASJ, 54, 905

Groh, J. H., Georgy, C., \& Ekström, S. 2013, A\&A, 558, LL1

Hachinger, S., Mazzali, P. A., Taubenberger, S., et al. 2012, MNRAS, 422, 70

Hamuy, M., Suntzeff, N. B., Heathcote, S. R., et al. 1994, PASP, 106, 566

Hamuy, M., Walker, A. R., Suntzeff, N. B., et al. 1992, PASP, 104, 533

Harkness, R. P., Wheeler, J. C., Margon, B., et al. 1987, ApJ, 317, 355

Hauschildt, P. H., \& Baron, E. 1999, JCoAM, 109, 41

Hauschildt, P. H., \& Baron, E. 2014, A\&A, 566, A89

Herbig, G. H. 1995, ARA\&A, 33, 19

Heydari-Malayeri, M., Grebel, E. K., Melnick, J., \& Jorda, L. 1993, A\&A, 278,11

Hill, G. J., Nicklas, H. E., MacQueen, P. J., et al. 1998, Proc. SPIE, 3355, 375

Hines, D. C., Rieke, G. H., Gordon, K. D., et al. 2004, ApJS, 154, 290

Hsiao, E. Y., Marion, G. H., Phillips, M. M., et al. 2013, ApJ, 766, 72

Hwang, U., Laming, J. M., Badenes, C., et al. 2004, ApJL, 615, L117

James, S., \& Baron, E. 2010, ApJ, 718, 957

Jeffery, D. J., \& Branch, D. 1990, in Supernovae, Jerusalem Winter School for Theoretical Physics, ed. J. C. Wheeler, T. Piran, \& S. Weinberg (Singapore: World Scientific), 149

Jewett, L., Cenko, S. B., Li, W., et al. 2012, CBET, 3037, 1

Jordi, K., Grebel, E. K., \& Ammon, K. 2006, A\&A, 460, 339

Kashikawa, N., Aoki, K., Asai, R., et al. 2002, PASJ, 54, 819

Kelly, P. L., Filippenko, A. V., Modjaz, M., \& Kocevski, D. 2014, ApJ, 789, 23

Kelly, P. L., \& Kirshner, R. P. 2012, ApJ, 759, 107

Kelly, P. L., Kirshner, R. P., \& Pahre, M. 2008, ApJ, 687, 1201

Ketchum, W., Baron, E., \& Branch, D. 2008, ApJ, 674, 371

Kirshner, R. P., Oke, J. B., Penston, M. V., \& Searle, L. 1973, ApJ, 185, 303

Kozasa, T., Hasegawa, H., \& Nomoto, K. 1991, A\&A, 249, 474

Krause, O., Birkmann, S. M., Usuda, T., et al. 2008, Sci, 320, 1195

Lazzati, D., Morsony, B. J., Blackwell, C. H., \& Begelman, M. C. 2012, ApJ, 750,68

Le Bertre, T., \& Lequeux, J. 1993, A\&A, 274, 909

Levesque, E. M., Soderberg, A. M., Foley, R. J., et al. 2010, ApJL, 709, L26

Li, C., Hillier, D. J., \& Dessart, L. 2012, MNRAS, 426, 1671

Li, H., \& McCray, R. 1992, ApJ, 387, 309

Lucy, L. B., Danziger, I. J., Gouiffes, C., \& Bouchet, P. 1989, in IAU Colloq. 120, Structure and Dynamics of the Interstellar Medium, ed. G. TenorioTagle, M. Moles, \& J. Melnick (Lecture Notes in Physics, Vol. 350; Berlin: Springer), 164
Maeda, K., Kawabata, K., Mazzali, P. A., et al. 2008, Sci, 319, 1220

Maeda, K., Nakamura, T., Nomoto, K., et al. 2002, ApJ, 565, 405 Margutti, R., Milisavljevic, D., Soderberg, A., et al. 2014, arXiv:1402.6344

Margutti, R., Soderberg, A. M., Chomiuk, L., et al. 2012, ApJ, 751, 134

Marion, G. H., Vinko, J., Kirshner, R. P., et al. 2014, ApJ, 781, 69

Matheson, T., Filippenko, A. V., Li, W., Leonard, D. C., \& Shields, J. C. 2001, AJ, 121,1648

Matheson, T., Garnavich, P. M., Stanek, K. Z., et al. 2003, ApJ, 599, 394

Matzner, C. D. 2003, MNRAS, 345, 575

Maund, J. R., Wheeler, J. C., Baade, D., et al. 2009, ApJ, 705, 1139

Maund, J. R., Wheeler, J. C., Patat, F., et al. 2007, MNRAS, 381, 201

Maurer, J. I., Mazzali, P. A., Deng, J., et al. 2010, MNRAS, 402, 161

Mazzali, P. A., Deng, J., Maeda, K., et al. 2002, ApJL, 572, L61

Mazzali, P. A., Kawabata, K. S., Maeda, K., et al. 2005, Sci, 308, 1284

Mazzali, P. A., Kawabata, K. S., Maeda, K., et al. 2007, ApJ, 670, 592

Mazzali, P. A., \& Lucy, L. B. 1998, MNRAS, 295, 428

Mazzali, P. A., Maurer, I., Stritzinger, M., et al. 2011, MNRAS, 416, 881

Mazzali, P. A., Maurer, I., Valenti, S., Kotak, R., \& Hunter, D. 2010, MNRAS, 408, 87

Mazzali, P. A., Nomoto, K., Maeda, K., et al. 2008a, in ASP Conf. Ser. 391, Hydrogen-Deficient Stars, ed. A. Werner \& T. Rauch (San Francisco, CA: ASP), 347

Mazzali, P. A., Nomoto, K., Patat, F., \& Maeda, K. 2001, ApJ, 559, 1047

Mazzali, P. A., Walker, E. S., Pian, E., et al. 2013, MNRAS, 432, 2463

Mazzali, P. A., Valenti, S., Della Valle, M., et al. 2008b, Sci, 321, 1185

Milisavljevic, D., \& Fesen, R. A. 2013, ApJ, 772, 134

Milisavljevic, D., Fesen, R. A., Gerardy, C. L., Kirshner, R. P., \& Challis, P. 2010, ApJ, 709, 1343

Milisavljevic, D., Fesen, R., Soderberg, A., et al. 2012, CBET, 3037, 2

Milisavljevic, D., Margutti, R., Crabtree, K. N., et al. 2014, ApJL, 782, L5 (M14)

Milisavljevic, D., Margutti, R., Soderberg, A. M., et al. 2013, ApJ, 767, 71

Miller, J. S., \& Stone, R. P. S. 1993, Lick Obs. Tech. Rep. 66 (Santa Cruz: Lick Obs.)

Minkowski, R. 1941, PASP, 53, 224

Modjaz, M., Blondin, S., Kirshner, R. P., et al. 2014, AJ, 147, 99

Modjaz, M., Kewley, L., Bloom, J. S., et al. 2011, ApJL, 731, L4

Modjaz, M., Kirshner, R. P., Blondin, S., Challis, P., \& Matheson, T. 2008, ApJL, 687, L9

Nakar, E., \& Sari, R. 2012, ApJ, 747, 88

Nomoto, K., Tominaga, N., Tanaka, M., \& Maeda, K. 2007, in AIP Conf. Proc. 924, The Multicolored Landscape of Compact Objects and Their Explosive Origins, ed. T. di Salvo, G. L. Israel, L. Piersant, L. Burderi, G. Matt, A. Tornambe, \& M. T. Menna (Melville, NY: AIP), 108

Nomoto, K. I., Iwamoto, K., \& Suzuki, T. 1995, PhR, 256, 173

Nota, A., Livio, M., Clampin, M., \& Schulte-Ladbeck, R. 1995, ApJ, 448, 788

Oke, J. B. 1990, AJ, 99, 1621

Oke, J. B., Cohen, J. G., Carr, M., et al. 1995, PASP, 107, 375

Oke, J. B., \& Searle, L. 1974, ARA\&A, 12, 315

Olivares, E. F., Greiner, J., Schady, P., et al. 2012, A\&A, 539, A76

Parrent, J., Branch, D., Troxel, M. A., et al. 2007, PASP, 119, 135

Patat, F., Cappellaro, E., Danziger, J., et al. 2001, ApJ, 555, 900

Pettini, M., \& Pagel, B. E. J. 2004, MNRAS, 348, L59

Pian, E., Mazzali, P. A., Masetti, N., et al. 2006, Natur, 442, 1011

Pignata, G., Stritzinger, M., Soderberg, A., et al. 2011, ApJ, 728, 14

Piro, A. L., \& Morozova, V. S. 2014, ApJL, 792, L11

Podsiadlowski, P., Joss, P. C., \& Hsu, J. J. L. 1992, ApJ, 391, 246

Podsiadlowski, P., Mazzali, P. A., Nomoto, K., Lazzati, D., \& Cappellaro, E. 2004, ApJL, 607, L17

Poole, T. S., Breeveld, A. A., Page, M. J., et al. 2008, MNRAS, 383, 627

Poznanski, D., Prochaska, J. X., \& Bloom, J. S. 2012, MNRAS, 426, 1465

Rest, A., Foley, R. J., Sinnott, B., et al. 2011, ApJ, 732, 3

Roming, P. W. A., Kennedy, T. E., Mason, K. O., et al. 2005, SSRv, 120, 95

Sahu, D. K., Tanaka, M., Anupama, G. C., Gurugubelli, U. K., \& Nomoto, K. 2009, ApJ, 697, 676

Sanders, N. E., Soderberg, A. M., Levesque, E. M., et al. 2012a, ApJ, 758, 132

Sanders, N. E., Soderberg, A. M., Valenti, S., et al. 2012b, ApJ, 756, 184

Schlafly, E. F., \& Finkbeiner, D. P. 2011, ApJ, 737, 103

Schmidt, G. D., Weymann, R. J., \& Foltz, C. B. 1989, PASP, 101, 713

Shklovskii, I. S. 1960, SvA, 4, 355

Simcoe, R. A., Burgasser, A. J., Bernstein, R. A., et al. 2008, Proc. SPIE, 7014, 70140U

Smartt, S. J. 2009, ARA\&A, 47, 63

Smith, N., Li, W., Filippenko, A. V., \& Chornock, R. 2011, MNRAS, 412, 1522

Soderberg, A. M., Chakraborti, S., Pignata, G., et al. 2010, Natur, 463, 513

Soderberg, A. M., Chevalier, R. A., Kulkarni, S. R., \& Frail, D. A. 2006a, ApJ, 651,1005 
Soderberg, A. M., Nakar, E., Berger, E., \& Kulkarni, S. R. 2006b, ApJ, 638, 930 Springob, C. M., Masters, K. L., Haynes, M. P., Giovanelli, R., \& Marinoni, C. 2009, ApJS, 182, 474

Spyromilio, J., Meikle, W. P. S., \& Allen, D. A. 1990, MNRAS, 242, 669

Stanek, K. Z., Matheson, T., Garnavich, P. M., et al. 2003, ApJL, 591, L17

Sutherland, P. G., \& Wheeler, J. C. 1984, ApJ, 280, 282

Swartz, D. A., Filippenko, A. V., Nomoto, K., \& Wheeler, J. C. 1993, ApJ, 411,313

Tan, J. C., Matzner, C. D., \& McKee, C. F. 2001, ApJ, 551, 946

Tanaka, M., Kawabata, K. S., Hattori, T., et al. 2012, ApJ, 754, 63

Tanaka, M., Kawabata, K. S., Maeda, K., Hattori, T., \& Nomoto, K. 2008, ApJ, 689, 1191

Taubenberger, S., Pastorello, A., Mazzali, P. A., et al. 2006, MNRAS, 371, 1459

Taubenberger, S., Valenti, S., Benetti, S., et al. 2009, MNRAS, 397, 677

Theureau, G., Bottinelli, L., Coudreau-Durand, N., et al. 1998, A\&AS, 130, 333

Thomas, R. C., Nugent, P. E., \& Meza, J. C. 2011, PASP, 123, 237

Turatto, M. 2003, in Supernovae and Gamma-Ray Bursters, ed. K. Weiler (Lecture Notes in Physics, Vol. 598; Berlin: Springer), 21

Turatto, M., Benetti, S., \& Cappellaro, E. 2003, in From Twilight to Highlight: The Physics of Supernovae, ed. W. Hillebrandt \& B. Leibundgut (Berlin: Springer), 200
Ugliano, M., Janka, H.-T., Marek, A., \& Arcones, A. 2012, ApJ, 757, 69

Valenti, S., Benetti, S., Cappellaro, E., et al. 2008a, MNRAS, 383, 1485

Valenti, S., Elias-Rosa, N., Taubenberger, S., et al. 2008b, ApJL, 673, L155

Valenti, S., Fraser, M., Benetti, S., et al. 2011, MNRAS, 416, 3138

Van Dyk, S. D., Li, W., \& Filippenko, A. V. 2003, PASP, 115, 1

Walker, E. S., Mazzali, P. A., Pian, E., et al. 2014, MNRAS, 442, 2768

Wang, L., \& Wheeler, J. C. 2008, ARA\&A, 46, 433

Wheeler, J. C., Harkness, R. P., Barker, E. S., Cochran, A. L., \& Wills, D. 1987, ApJL, 313, L69

Wheeler, J. C., Höflich, P., Harkness, R. P., \& Spyromilio, J. 1998, ApJ, 496, 908

Wheeler, J. C., Johnson, V., \& Clocchiatti, A. 2014, arXiv:1411.5975

Woosley, S. E., \& Bloom, J. S. 2006, ARA\&A, 44, 507

Woosley, S. E., Langer, N., \& Weaver, T. A. 1993, ApJ, 411, 823

Xu, D., Zhang, J.-J., Chen, J., et al. 2012, ATel, 3922, 1

Yoon, S.-C., Woosley, S. E., \& Langer, N. 2010, ApJ, 725, 940

Young, D. R., Smartt, S. J., Valenti, S., et al. 2010, A\&A, 512, A70

Zaritsky, D., Kennicutt, R. C., Jr., \& Huchra, J. P. 1994, ApJ, 420, 87

Zurita, A., \& Bresolin, F. 2012, MNRAS, 427, 1463 